\title{
Mathematical Modelling of Tumour Invasion and Metastasis
}

\author{
A. R. A. ANDERSON ${ }^{a, *}$, M. A. J. CHAPLAIN ${ }^{a, \dagger}$, E. L. NEWMAN ${ }^{b}$, R. J. C. STEELE ${ }^{b}$ and A. M. THOMPSON ${ }^{b}$ \\ ${ }^{a}$ Department of Mathematics, University of Dundee, Dundee DDI 4HN, Scotland; ${ }^{b}$ Department of Surgery and Molecular Oncology, \\ University of Dundee, Ninewells Hospital and Medical School, Dundee DDI 957
}

(Received April 1999; In final form 10 August 1999)

\begin{abstract}
In this paper we present two types of mathematical model which describe the invasion of host tissue by tumour cells. In the models, we focus on three key variables implicated in the invasion process, namely, tumour cells, host tissue (extracellular matrix) and matrixdegradative enzymes associated with the tumour cells. The first model focusses on the macro-scale structure (cell population level) and considers the tumour as a single mass. The mathematical model consists of a system of partial differential equations describing the production and/or activation of degradative enzymes by the tumour cells, the degradation of the matrix and the migratory response of the tumour cells. Numerical simulations are presented in one and two space dimensions and compared qualitatively with experimental and clinical observations. The second type of model focusses on the micro-scale (individual cell) level and uses a discrete technique developed in previous models of angiogenesis. This technique enables one to model migration and invasion at the level of individual cells and hence it is possible to examine the implications of metastatic spread. Finally, the results of the models are compared with actual clinical observations and the implications of the model for improved surgical treatment of patients are considered.
\end{abstract}

Keywords: Tumour cells, invasion, metastasis, extracellular matrix, matrix degrading enzyme

\section{INTRODUCTION}

The development of a primary solid tumour (e.g., a carcinoma) begins with a single normal cell becoming transformed as a result of mutations in certain key genes. This transformed cell differs from a normal one in several ways, one of the most notable being its escape from the body's homeostatic mechanisms, leading to inappropriate proliferation. An individual tumour cell has the potential, over successive divisions, to develop into a cluster (or nodule) of tumour cells. Further growth and proliferation leads to the development of an avascular tumour consisting of approximately $10^{6}$ cells. This cannot grow any further, owing to its dependence on diffusion as the only means of receiving nutrients and removing waste products. For any further development to occur the tumour must initiate angiogenesis - the recruitment

\footnotetext{
${ }^{*}$ Corresponding Author: Tel: (+44) 01382 344462; Fax: (+44) 01382 345516; E-mail: anderson@mcs.dundee.ac.uk

†Tel: (+44) 01382 345369; Fax: (+44) 01382 345516; E-mail: chaplain@mcs.dundee.ac.uk
} 
of blood vessels. The tumour cells first secrete angiogenic factors which in turn induce endothelial cells in a neighbouring blood vessel to degrade their basal lamina and begin to migrate towards the tumour. As it migrates, the endothelium begins to form sprouts which can then form loops and branches through which blood circulates. From these branches more sprouts form and the whole process repeats forming a capillary network which eventually connects with the tumour, completing angiogenesis and supplying the tumour with the nutrients it needs to grow further. There is now also the possibility of tumour cells finding their way into the circulation and being deposited in distant sites in the body, resulting in metastasis. The complete process of metastasis involves several sequential steps, each of which must be successfully completed by cells of the primary tumour before a secondary tumour (a metastasis) is formed. A summary of the key stages of the metastatic cascade is as follows:

- cancer cells escape from the primary tumour;

- they locally degrade the surrounding tissue and continue migration;

- they enter the lymphatic or blood circulation system (intravasation);

- they must survive their journey in the circulation system;

- they must escape from the blood circulation (extravasation);

- the cancer cells (from the primary tumour) must then establish a new colony in distant organs;

- the new colony of cells must then begin to grow to form a secondary tumour in the new organ.

A crucial part of the invasive/metastatic process is the ability of the cancer cells to degrade the surrounding tissue or extracellular matrix (ECM) (Liotta et al., 1983; Stetler-Stevenson et al., 1993; Lawrence and Steeg, 1996). This is a complex mixture of macromolecules, some of which, like the collagens, are believed to play a structural role and others, such as laminin, fibronectin and vitronectin, are important for cell adhesion, spreading and motility. We note that all of these macromolecules are bound within the tissue i.e. they are non-diffusible. The ECM can also sequester growth factors and itself be degraded to release fragments which can have growth-promoting activity. Thus, while ECM may have to be physically removed in order to allow a tumour to spread or intraor extravasate, its degradation may in addition have biological effects on tumour cells.

A number of matrix degradative enzymes (MDEs) such as the plasminogen activator (PA) system and the large family of matrix metalloproteinases (MMPs) have been described (Mignatti and Rifkin, 1993; Matrisian, 1992; Thorgeirsson et al, 1994). While no MDE is completely specific for one element of the ECM, some broad preferences are expressed, for example the gelatinases (two members of the MMP family) preferentially cleave the laminar collagens IV and V and denatured fibrillar collagens I, II and III but can also digest vitronectin and laminin, at least in vitro (reviewed in Yu et al., 1998). Both PAs and the MMPs have been repeatedly implicated in all of the above steps of tumour invasion and metastasis (Ahmad et al., 1998; Bafetti et al., 1998; Brown, 1998; Chambers and Matrisian, 1997; Kim et al., 1998; Itoh et al., 1998; Koshiba et al., 1998; Parson et al., 1997; Sehgal et al., 1998; StetlerStevenson et al., 1996; Zeng and Guillem, 1998). Regulation of matrix-degradative activity is highly complex. In both these enzyme systems (PAs/MMPs) there exist several endogenous inhibitors (Beattie and Smyth, 1998; Kleiner and Stetler-Stevenson, 1993; Stetler-Stevenson et al., 1989), and the enzymes are often secreted as inactive precursors which must themselves be partially degraded to reach full activity. More than one cell type may be involved in the activation of any one enzyme (Kleiner and StetlerStevenson, 1993).

Over the last ten years or so many mathematical models of tumour growth, both temporal and spatiotemporal, have appeared in the research literature (see Chaplain, 1996, for a review of many of these). Much of the experimental data that exist on the growth kinetics of avascular tumours have been incorporated into mathematical models using various growth laws such as Gompertzian growth, logistic growth and exponential growth, to name but a few (see, for example, Wheldon, 1986; Retsky et al., 1990; Marusic et al., 1994; and references therein). Modelling 
of the important process of tumour-induced angiogenesis and capillary network formation has also been undertaken (Chaplain and Stuart, 1993; Chaplain, 1995; Anderson and Chaplain, 1998). Deterministic reaction-diffusion equations have been used to model the spatial spread of tumours both at an early stage in its growth (Sherratt and Nowak, 1992) and at the later invasive stage (Orme and Chaplain, 1996; Gatenby, 1996; Perumpanani et al., 1996). Modelling of a related phenomenon, embryonic implantation involving invading trophoblast cells, using a reactiondiffusion approach has also been carried out (Byme et al., 1999). Typical solutions observed in all these models (Orme and Chaplain, 1996; Gatenby, 1996; Perumpanani et al., 1996; Byrne et al., 1999) appear as invading travelling waves of cancer cells. An alternative framework is to adopt a continuum/solid mechanics approach or a mechano-chemical modelling approach (Chaplain and Sleeman, 1993; Tracqui, 1995) and to consider physical pressure and forces between cells and matrix. Whilst these models are able to capture the tumour structure at the tissue level, they fail to describe the tumour at the cellular level and subsequently the subcellular level. On the other hand, cellular automata models provide such a description and allow a more realistic stochastic approach at both the cellular (Kim- mel and Axelrod, 1991; Smolle and Stettner, 1993; Qi et al., 1993) and subcellular levels (Düchting, 1990a,b; 1992; Düchting et al., 1996).

The models presented in this paper are of two types: a continuum, deterministic model (based on a system of reaction-diffusion-chemotaxis equations) and a discrete, quasi-stochastic model (based on a biased random-walk model). We choose to focus on three key variables involved in tumour cell invasion, thereby producing a minimal model, namely; tumour cells, ECM and MDEs. Initially we derive a system of coupled nonlinear partial differential equations, using conservation laws, to model tumour invasion of surrounding tissue. Numerical solutions for this system in both one and two dimensions will be presented, thus allowing the macroscopic dynamics of invasion to be discussed. From a discretised form of these partial differential equations, we derive a discrete biased random-walk model which enables the migration and proliferation of individual cells to be considered.

The main aims of this paper are (i) to lay the foundations for developing quantitative mathematical models of tumour invasion; (ii) to investigate the importance of ECM-tumour interactions in governing the migration of tumour cells and (iii) to make predictions about the metastatic ability of tumour cells. For example, by considering the cells as discrete individuals we can estimate, for a given initial tumour, how far it will invade and the numbers of cells that migrate outwith the main bulk of the tumour and thus allow for both qualitative and quantitative comparisons with experimental and clinical data. From the clinical perspective, it is the escape of tumour cells beyond the boundary of detectable tumour mass (which may be resected surgically), that gives rise to the serious problems of local and distant recurrence.

The layout of the paper is therefore as follows: in the next section, we formulate the continuum model of invasion based on a system of partial differential equations. In Section 3 we present the results of numerical simulations of this model in 1 and 2 dimensions. In Section 4 we derive the discrete biased random walk model (based on the model of Section 2) and present the results of the discrete simulations in Section 5. Finally in Section 6 we discuss the clinical implications of the results of the model and make some concluding remarks.

\section{THE CONTINUOUS MATHEMATICAL MODEL}

We will base our mathematical model on generic solid tumour growth, which for simplicity we will assume is at the avascular stage. Whilst most tumours are asymptomatic at this state, it is still possible for cells to escape and migrate to the lymph nodes and for the more aggressive tumours to invade. The model may be extended to incorporate interactions between the tumour cells and blood vessels, thereby modelling angiogenesis and vascular growth. However since one of the aims of the paper is to focus solely on the 
interactions between the tumour and the surrounding tissue we do not attempt to model interactions between the tumour and the vasculature. In principle, our model can be extended to include such interactions and the general form of our model will be the same for both invading vascular and avascular tumours. In the model we therefore consider the three variables; tumour cell density (denoted by $n$ ), MDE concentration (denoted by $m$ ) and ECM density (denoted by $f$ ). Each of the three variables $(n, m, f$ ) is a function of the spatial variable $\mathbf{x}$ and time $t$.

Most of the macromolecules of the ECM which are important for cell adhesion, spreading and motility (e.g. fibronectin, laminin, and vitronectin) are fixed or bound to the surrounding tissue. As already discussed in the introduction, MDEs are important at many stages of tumour growth, invasion and metastasis, and the manner in which they interact with inhibitors, growth factors and tumour cells is very complex. However, it is well known that the tumour cells produce MDEs which degrade the ECM locally. As well as making space into which tumour cells may move by simple diffusion (random motility), we assume that this also results in a gradient of these bound cell-adhesion molecules, such as fibronectin. Therefore while the ECM may constitute a barrier to normal cell movement, it also provides a substrate to which cells may adhere and upon which they may move. Most mammalian cell types require at least some elements of the ECM to be present for growth and survival and will indeed migrate up a gradient of bound (i.e. non-diffusible) cell adhesion molecules in culture in vitro (Carter, 1965; Quigley et al., 1983; Lacovara et al., 1984; McCarthy and Furcht, 1984; Klominek et al., 1993; Lawrence and Steeg, 1996).

By definition, haptotaxis is the directed migratory response of cells to gradients of fixed or bound chemicals (i.e. non-diffusible chemicals). While it has not yet been explicitly demonstrated that haptotaxis occurs in an in vivo situation, given the structure of human tissue, it is not unreasonable to assume that haptotaxis is a major component of directed movement in tumour cell invasion. Indeed, there has been much recent effort to characterise such directed movement (Klominek et al., 1993; Lawrence and Steeg, 1996). We therefore refer to this directed movement of tumour cells in our model as haptotaxis i.e. a response to gradients of bound macromolecules such as fibronectin. To incorporate this response in our mathematical model, we take the haptotactic flux to be $\mathbf{J}_{\text {hapto }}=\chi n \nabla f$, where $\chi>0$ is the (constant) haptotactic coefficient.

As mentioned above, the only other contribution to tumour cell motility in our model is assumed to be random motion. This approach permits us to investigate cell-matrix interactions in isolation (i.e. in the absence of cell proliferation). To describe the random motility of the tumour cells we assume a fiux of the form $\mathbf{J}_{\text {random }}=-D(f, m) \nabla n$, where $D(f, m)$ may be a constant or a function of either the MDE or ECM concentration, the latter cases representing a chemokinetic response i.e., increased random motility will be observed for regions of high MDE/ECM concentration.

To enable us to focus entirely on the cell-matrix interactions and how these interactions affect tumour cell migration, we do not consider any proliferation of tumour cells in our partial differential equation model. However tumour cell proliferation will be included in the discrete model in Section 4. The conservation equation for the tumour cell density $n$ is therefore given by

$$
\frac{\partial n}{\partial t}+\nabla \cdot\left(\mathbf{J}_{\text {rand }}+\mathbf{J}_{\text {hapto }}\right)=0
$$

and hence the partial differential equation governing tumour cell motion (in the absence of cell proliferation) is,

$$
\frac{\partial n}{\partial t}=\nabla \cdot(D(f, m) \nabla n)-\chi \nabla \cdot(n \nabla f) .
$$

For the initial simulations given in the next section we chose $D(f, m)=D_{n}$, a constant, the tumour cell random motility coefficient.

The ECM is known to contain many macromolecules, including fibronectin, laminin and collagen, which can be degraded by MDEs (StetlerStevenson et al., 1996; Chambers and Matrisian, 1997). We assume that the MDEs degrade ECM upon contact and hence the degradation process is modelled 
by the following simple equation:

$$
\frac{\partial f}{\partial t}=-\delta m f
$$

where $\delta$ is a positive constant.

Active MDEs are produced (or activated) by the tumour cells, diffuse throughout the tissue and undergo some form of decay (either passive or active). The equation governing the evolution of MDE concentration is therefore given by:

$$
\frac{\partial m}{\partial t}=D_{m} \nabla^{2} m+g(n, m)-h(n, m, f)
$$

where $D_{m}$ is a positive constant, the MDE diffusion coefficient, $g$ is a function modelling the production of active MDEs by the tumour cells and $h$ is a function modelling the MDE decay. For simplicity we assume that there is a linear relationship between the density of tumour cells and the level of active MDEs in the surrounding tissues (regardless of the amount of enzyme precursors secreted and the presence of endogenous inhibitors) and so initially these functions were taken to be $g=\mu n$ (MDE production by the tumour cells) and $h=\lambda m$ (natural decay), respectively. Other functional forms for $h$ were also tried (see Section 3.1 for details).

Hence the complete system of equations describing the :eractions of the tumour cells, ECM and MDEs as dutailed in the previous paragraphs is

$$
\begin{aligned}
& \frac{\partial n}{\partial t}=\overbrace{D_{n} \nabla^{2} n}^{\text {random motility }}-\overbrace{\chi \nabla \cdot(n \nabla f)}^{\text {haptotaxis }}, \\
& \frac{\partial f}{\partial t}=-\overbrace{\delta m f}^{\text {degradation }}, \\
& \frac{\partial m}{\partial t}=\overbrace{D_{m} \nabla^{2} m}^{\text {diffusion }}+\overbrace{\mu n}^{\text {production }}-\overbrace{\lambda m}^{\text {decay }} .
\end{aligned}
$$

This system is considered to hold on some spatial domain $\Omega$ (a region of tissue) with appropriate initial conditions for each variable. We assume that the tumour cells, and consequently the MDEs, remain within the domain of tissue under consideration and therefore no-flux boundary conditions are imposed on $\partial \Omega$, the boundary of $\Omega$.
In order to solve the system numerically, we first of all non-dimensionalise the equations in the standard way. We rescale distance with an appropriate length scale $L$ (e.g. the maximum invasion distance of the cancer cells at this early stage of invasion $0.1-1 \mathrm{~cm}$ ), time with $\tau=L^{2} / D$ (where $D$ is a reference chemical diffusion coefficient $\sim 10^{-6} \mathrm{~cm}^{2} \mathrm{~s}^{-1}$, Bray, 1992), tumour cell density with $n_{0}$, ECM density with $f_{0}$ and MDE concentration with $m_{0}$ (where $n_{0}, f_{0}, m_{0}$ are appropriate reference variables). Therefore setting

$$
\tilde{n}=\frac{n}{n_{0}}, \quad \tilde{f}=\frac{f}{f_{0}}, \quad \tilde{m}=\frac{m}{m_{0}}, \quad \tilde{x}=\frac{x}{L}, \quad \tilde{t}=\frac{t}{\tau}
$$

in (4) and dropping the tildes for notational convenience, we obtain the scaled system of equations:

$$
\begin{aligned}
& \frac{\partial n}{\partial t}=\overbrace{d_{n} \nabla^{2} n}^{\text {random motility }}-\overbrace{\gamma \nabla \cdot(n \nabla f),}^{\text {haptotaxis }}, \\
& \frac{\partial f}{\partial t}=-\overbrace{\eta m f}^{\text {degradation }}, \\
& \frac{\partial m}{\partial t}=\overbrace{d_{m} \nabla^{2} m}^{\text {diffusion }}+\overbrace{\alpha n}^{\text {production }}-\overbrace{\beta m,}^{\text {decay }}
\end{aligned}
$$

where $d_{n}=D_{n} / D, \quad \gamma=\chi f_{0} / D, \eta=\tau m_{0} \delta, \quad d_{m}=$ $D_{m} / D, \alpha=\tau \mu n_{0} / m_{0}, \beta=\tau \lambda$. The cell motility parameter $D_{n} \sim 10^{-10} \mathrm{~cm}^{2} \mathrm{~s}^{-1}$ was estimated from available experimental evidence (Bray, 1992). The haptotactic parameter $\chi \sim 2600 \mathrm{~cm}^{2} \mathrm{~s}^{-1} \mathrm{M}^{-1}$ was estimated to be in line with that calculated in Anderson and Chaplain (1998) and the parameter $f_{0} \sim$ $10^{-8}-10^{-11} \mathrm{M}$ was taken from the experiments of Terranova et al., (1985). We took $D_{m}$ to be in the range $10^{-8}-10^{-10} \mathrm{~cm}^{2} \mathrm{~s}^{-1}$. Estimates for the kinetic parameters $\mu, \lambda, \delta$ were not available since these are very difficult to obtain experimentally.

The zero-flux boundary conditions:

$$
\zeta \cdot\left(-d_{n} \nabla n+n \gamma \nabla f\right)=0
$$

for the cells and

$$
\underline{\zeta} \cdot\left(-d_{m} \nabla m\right)=0,
$$

for the MDEs are imposed on the boundaries of the domain where $\zeta$ is an appropriate outward unit normal 
vector. In one space dimension, the scaled domain is the unit interval $[0,1]$, while in two space dimensions, the scaled domain is the unit square $[0,1] \times[0,1]$.

Initially we assume that there is a nodule of cells already present and in one dimension that the tumour is centred around $x=0$ with $n$ having the initial density distribution,

$$
n(x, 0)= \begin{cases}\exp \left(-x^{2} / \varepsilon\right), & x \in[0,0.25], \\ 0, & x \in(0.25,1]\end{cases}
$$

where $\varepsilon$ is a positive constant. The initial tumour density in two dimensions has a similar form, but
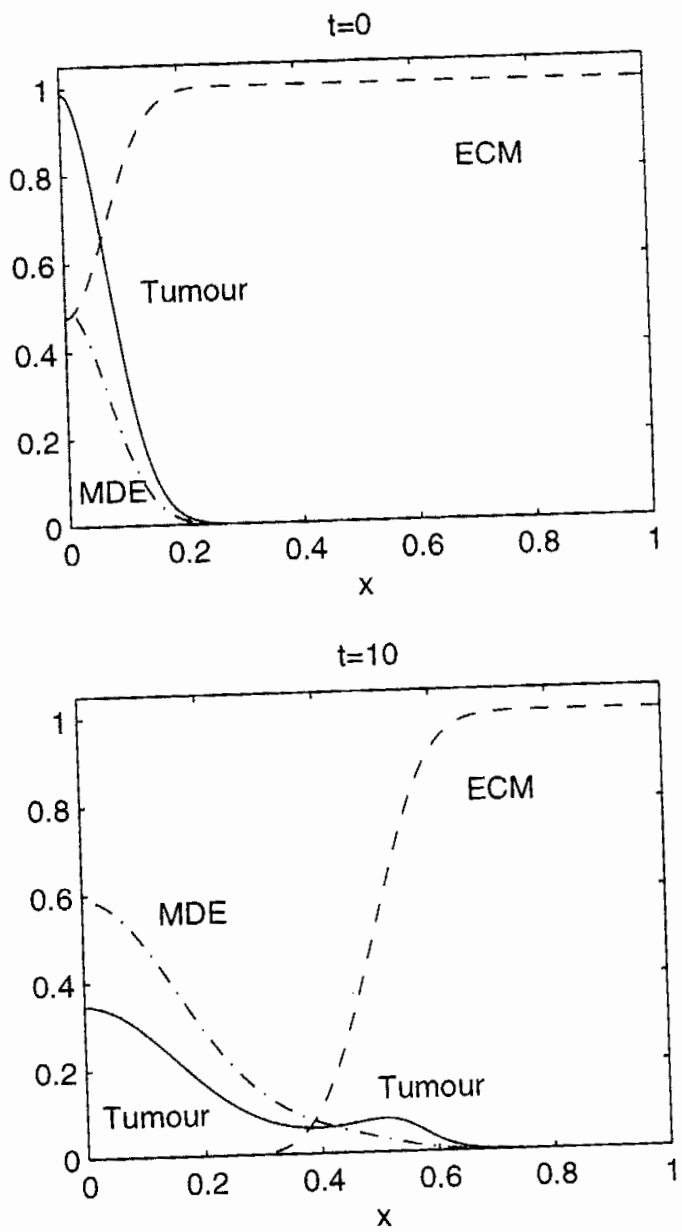

is centred on $(0.5,0.5)$ i.e.

$$
n(x, y, 0)= \begin{cases}\exp \left(-r^{2} / \varepsilon\right), & r \in[0,0.1], \\ 0, & r \in(0.1,1]\end{cases}
$$

where $\varepsilon$ is as above and $r^{2}=(x-0.5)^{2}+(y-0.5)^{2}$.

For both the one and two dimensional results we assume that the tumour has already degraded some of its surrounding tissue and hence we take the initial profile of ECM to be $f(\boldsymbol{x}, 0)=1-0.5 n(\boldsymbol{x}, 0)$. Finally, we assume that the initial MDE concentration profile is proportional to the initial tumour cell density and take $m(\boldsymbol{x}, 0)=0.5 n(\boldsymbol{x}, 0)$. These initial conditions are illustrated graphically at $t=0$ in each of the figures in the following section:
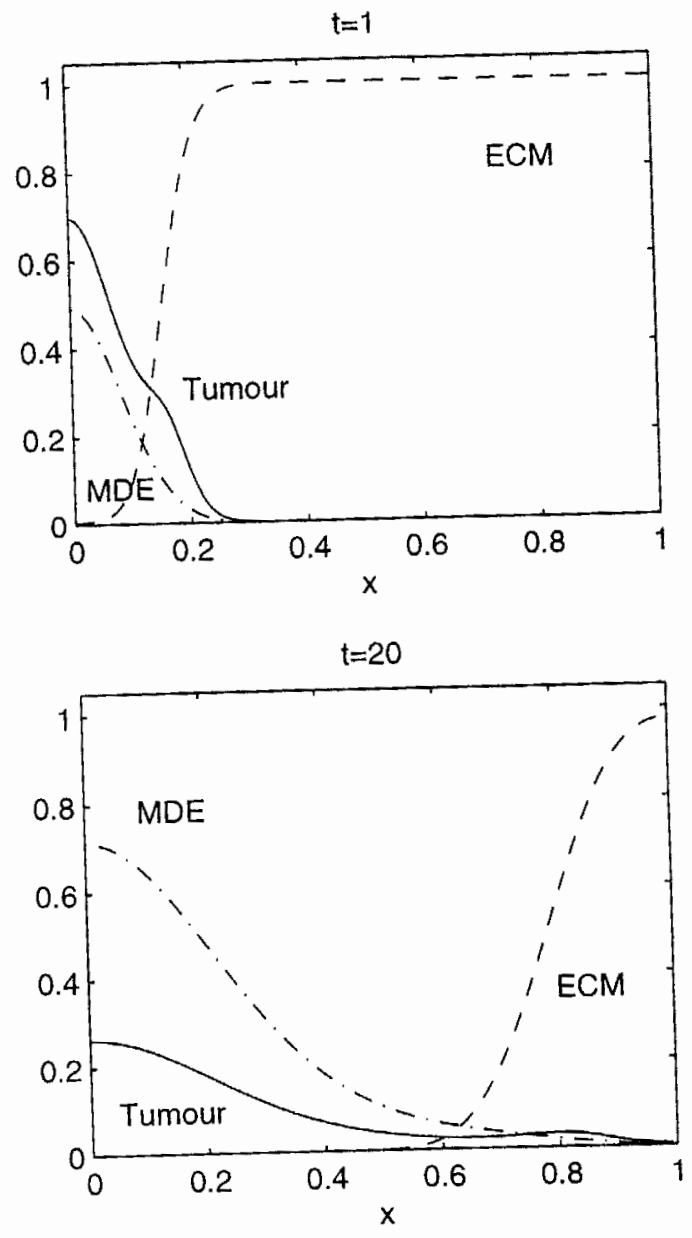

FIGURE 1 One dimensional numerical solution of the system (5) with constant tumour cell diffusion showing the cell density, MDE

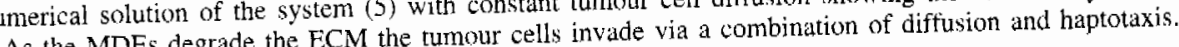

concentration and ECM density. As the MDEs degrade the ECM the turnour cels 

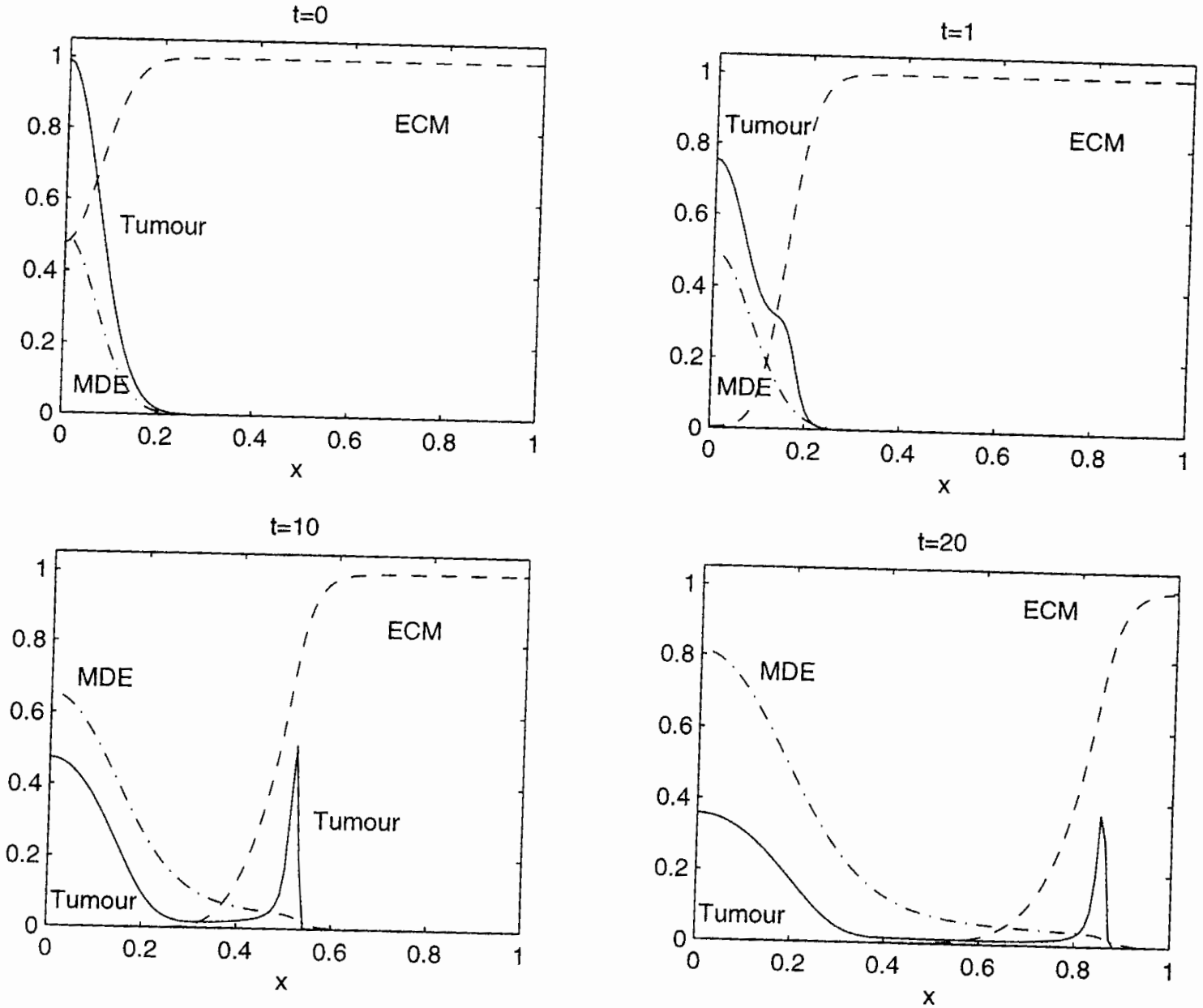

FIGL $\leq E 2$ One dimensional numerical solution of the system (5) with iumour cell diffusion dependent on MDE concentration. As the haptotaxis.
. ECM, the tumour ceils invade and form two distinct clusters, those mainly driven by diffusion and those driven by
.

\section{NUMERICAL SIMULATIONS}

\subsection{One dimensional results}

The following numerical results were obtained using the NAG routine D03PCF which implements the method of lines and Gear's Method. In the following simulations, the parameter values used were as follows: $d_{r_{c}}=0.001, d_{m}=0.001, \gamma=0.005, \eta=10$, $\alpha=0.1, \beta=0$ and $\varepsilon=0.01$.

Figure 1 shows four snapshots in time of the tumour cell density, ECM density, and MDE concentration. The ECM profile shows clearly the degradation by the MDEs. The tumour density distribution at $t=1$ shows that a small cluster of cells has built up at the leading edge of the tumour due to haptotactic migration. As time evolves this cluster of cells migrates further from the main body of the tumour, which continues to invade the ECM but at a slower rate. These figures indicate that the initial cluster of tumour cells may be able to break into two separate clusters. This becomes even more apparent in subsequent figures.

In the next simulation in Figure 2, we consider the effect of nonlinear diffusion on the invasion of the tumour cells by taking $D(f, m)=d_{n} m$. This represents a chemokinetic response of the tumour cells to MDE concentration where we make the simple 

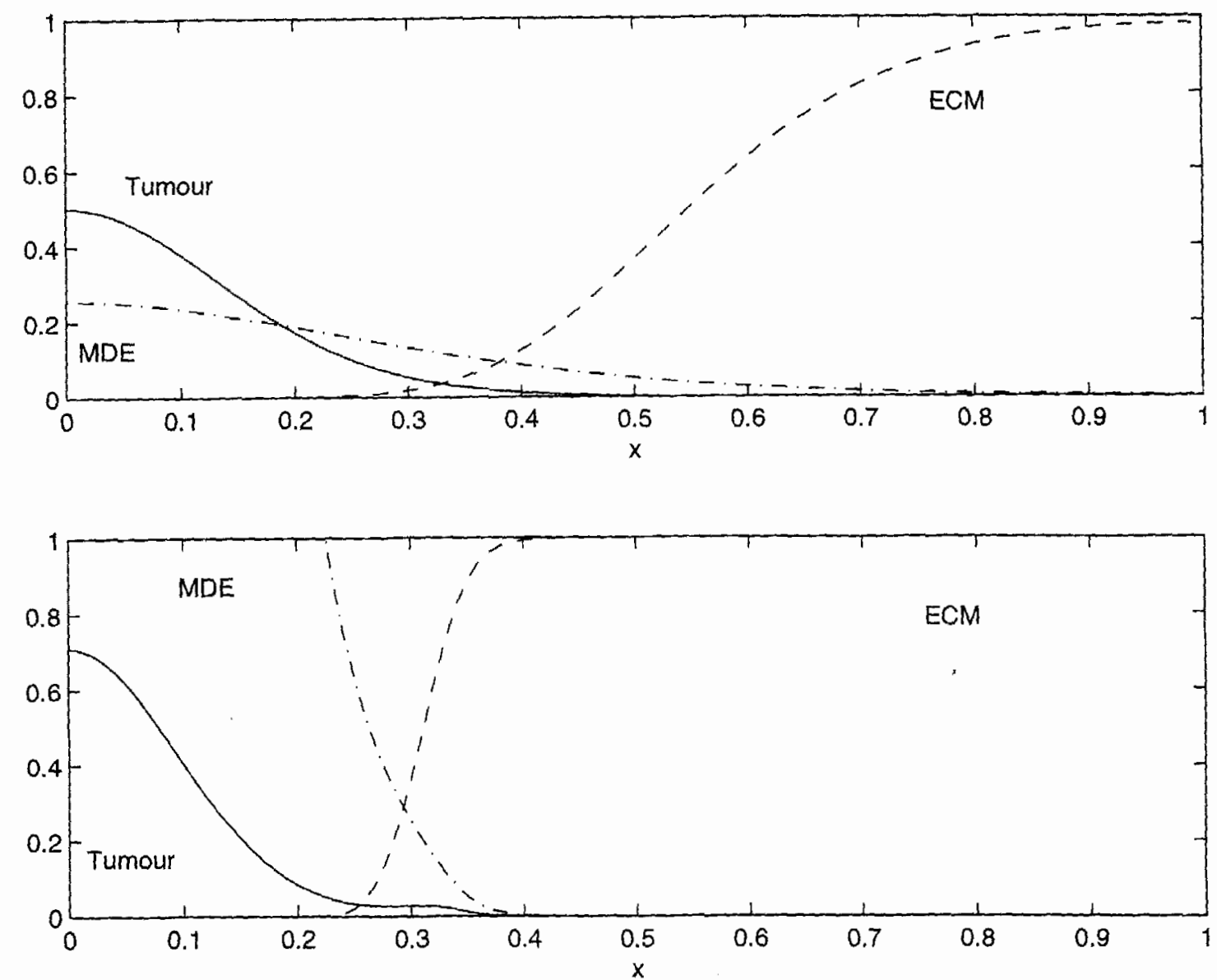

FIGURE 3 One dimensional numerical solutions of the system (5), with parameter values as in Figure 1 (unless otherwise stated), showing the cell density, MDE concentration and ECM density. (a) (top) shows the effect of increasing $d_{m}=0.01$ at $t=5$ and in (b) (bottom) the effect of increasing $\alpha=10$ at $t=2$.

assumption that the tumour cell random motility is directly proportional to MDE concentration i.e. where there is a high MDE concentration there is high random cell motility. Using the same parameters as in Figure 1, the four snapshots in Figure 2 were produced. Whilst the MDE and ECM concentration profiles closely resemble those obtained in Figure 1, the tumour density distribution has changed considerably. By $t=1$ we again see a build up of tumour cells at the leading edge, more pronounced than in Figure 1, which then breaks away from the main body of the tumour. This results in two quite distinct clusters, one of which migrates much further into the ECM. The main body of the tumour however, invades more slowly than was observed in Figure 1. If a small cluster of cells breaks away from the main body of the tumour, there is then the potential for these cells to intravasate any neighbouring vessels and start the metastatic cascade. Also if the main body of the tumour were to be surgically removed (resected), the smaller cluster of cells that has invaded further into the ECM may go unnoticed by the surgeon and lead to a possible recurrence. These results indicate the importance of haptotaxis as a mechanism for invasion and implicate its role in the metastatic cascade.

We now investigate the effect that changing various parameter values has on the solution. In particular we consider the effect of increasing the MDE diffusion coefficient $d_{m}$, MDE production rate $\alpha$ and the tumour cell haptotactic coefficient $\gamma$. 

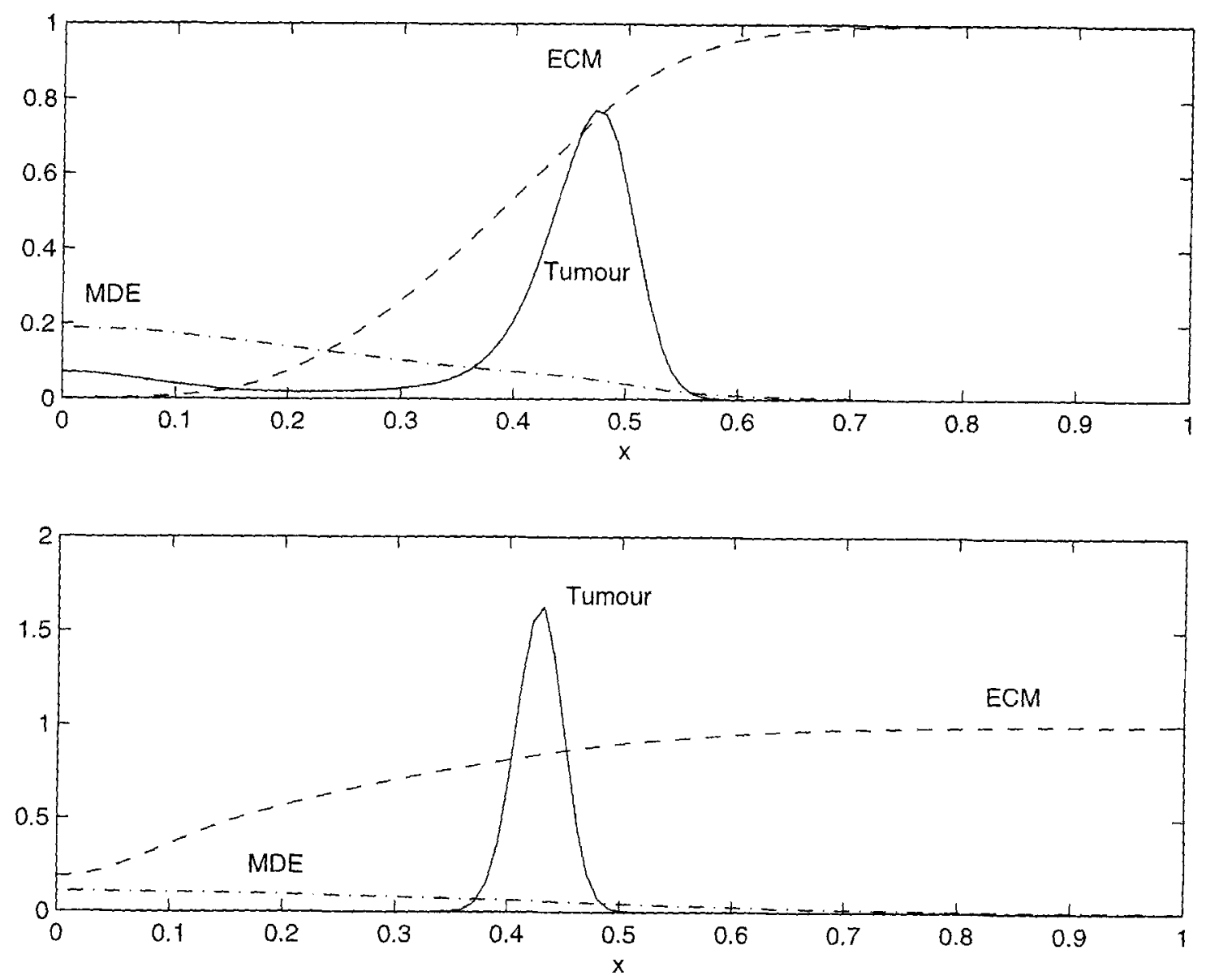

FIGURE 4 One dimensional numerical solutions of the system (5), with parameter values as in Figure 1 (unless otherwise stated), showing the cell density, MDE concentration and ECM density. (a) (top) shows the effect of increasing $d_{m}$ and $\gamma$ by a factor of 10 $\left(d_{m}=0.01, \gamma=0.05\right)$ at $t=2.5$ and in (b) (bottom) the effect of increasing these variables by a factor of $100\left(d_{m}=0.1, \gamma=0.5\right)$ at $t=0.5$.

In Figure 3(a) we show the effect of increasing the diffusion coefficient of the MDEs by a factor of 10 i.e. $d_{m}=0.01$ (all other parameter values unchanged from Figure 1). While in Figure 3(b) we increase the value of $\alpha$ by a factor of 100 i.e. $\alpha=10$, representing increased MDE production by the tumour cells (all other parameter values unchanged from Figure 1). In both cases, we can see that there has been more degradation of the matrix due to the fact that the MDE has either spread into the domain more rapidly (Figure 3a) or has been produced in greater quantity (Figure 3b). In each case the tumour cells remain more localised and do not invade the tissue as much.

In Figures 4(a) and (b) we show the effect of increasing both the MDE diffusion coefficient and the tumour cell haptotactic coefficient by factors of 10 and 100 respectively (all other parameter values unchanged from Figure 1). In Figure 4(a) $\left(d_{m}=0.01, \gamma=0.05\right)$ we can see that there is a larger proportion of tumour cells breaking away from the initial mass and invading the tissue compared with Figure (1). In Figure 4(b) $\left(d_{m}=0.1, \gamma=0.5\right)$ this effect has been accentuated even more as almost all the tumour cells have invaded the tissue, being driven mainly by haptotaxis.

In Figures 5(a) and 5(b) we have increased $\gamma$ by a factor of 10 and 100 respectively (all other parameters remain unchanged from Figure 1). These figures show the importance of tumour-matrix interactions and haptotaxis. As $\gamma$ is increased, a larger 

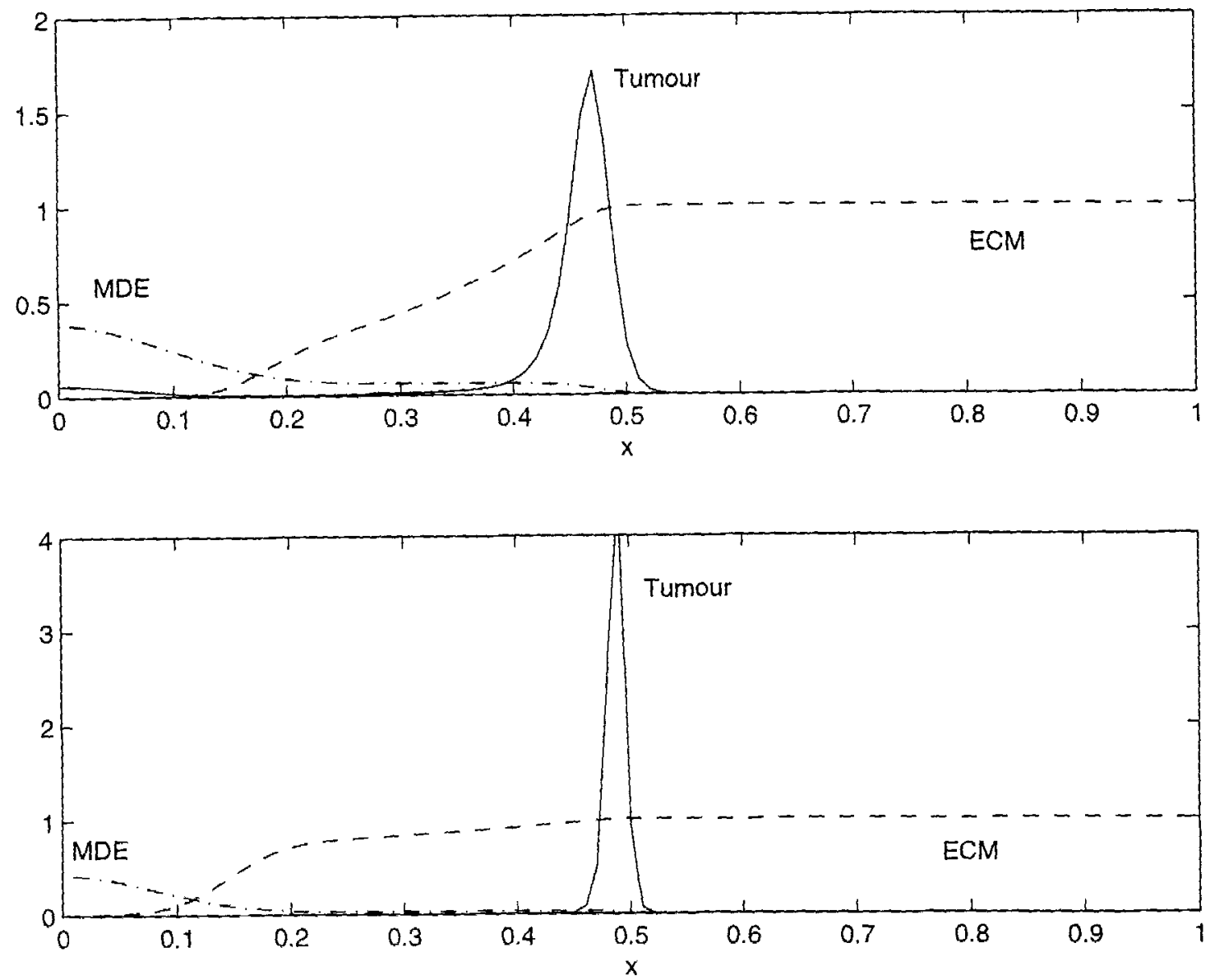

FIGURE 5 One dimensional numerical solutions of the system (5), with parameter values as in Figure I (unless otherwise stated), showing the cell density, MDE concentration and ECM density. (a) (top) shows the effect of increasing $\gamma$ by a factor of $10(\gamma=0.05)$ at $t=2.5$ and (b) (bottom) shows the effect of increasing $\gamma$ by a factor of $100(\gamma=0.5)$ at $t=1$.

proportion of the tumour cells invade the tissue, driven forward by haptotaxis and the gradients in the ECM. Indeed from Figure 5(b) we can see that almost all the tumour cells are invading in a pulse-like travelling wave.

Having examined the effect of different parameter values on the dynamics of the system, we now consider different functional forms for the MDE decay term, $h(m, n, f)$, since the exact dynamics of the $\mathrm{MDE} / \mathrm{ECM}$ and MDE/tumour cell interactions are not known. However, we do know that certain inhibitors (e.g. Tissue Inhibiting Metalloproteases, TIMPs) are produced within the ECM and that there will be some natural decay of MDEs. We have therefore chosen the following three functional forms: (i) $h=\beta m$, (ii) $h=$ $\beta m f$ and (iii) $h=\beta m(1-f)$, where $\beta$ is a positive constant. The biological interpretation for these forms is (i) natural decay, (ii) decay proportional to ECM density, modelling the assumption that production of MDE inhibitors (e.g. TIMPs) is directly proportional to the underlying ECM density and (iii) decay inversely proportional to ECM density, modelling the assumption that regions of higher ECM density allows for more MDE to bind there and degrade it.

Figures $6(a-c)$ show plots of the tumour cell density, MDE concentration and ECM density respectively, at $t=10$ with the same parameter values as for Figure 1 i.e. $d_{n}=0.001, d_{m}=0.001, \gamma=0.005$, $\eta=10, \alpha=0.1$ and the additional parameter $\beta=0.5$. Within each plot are four curves, one for $h=0$ 


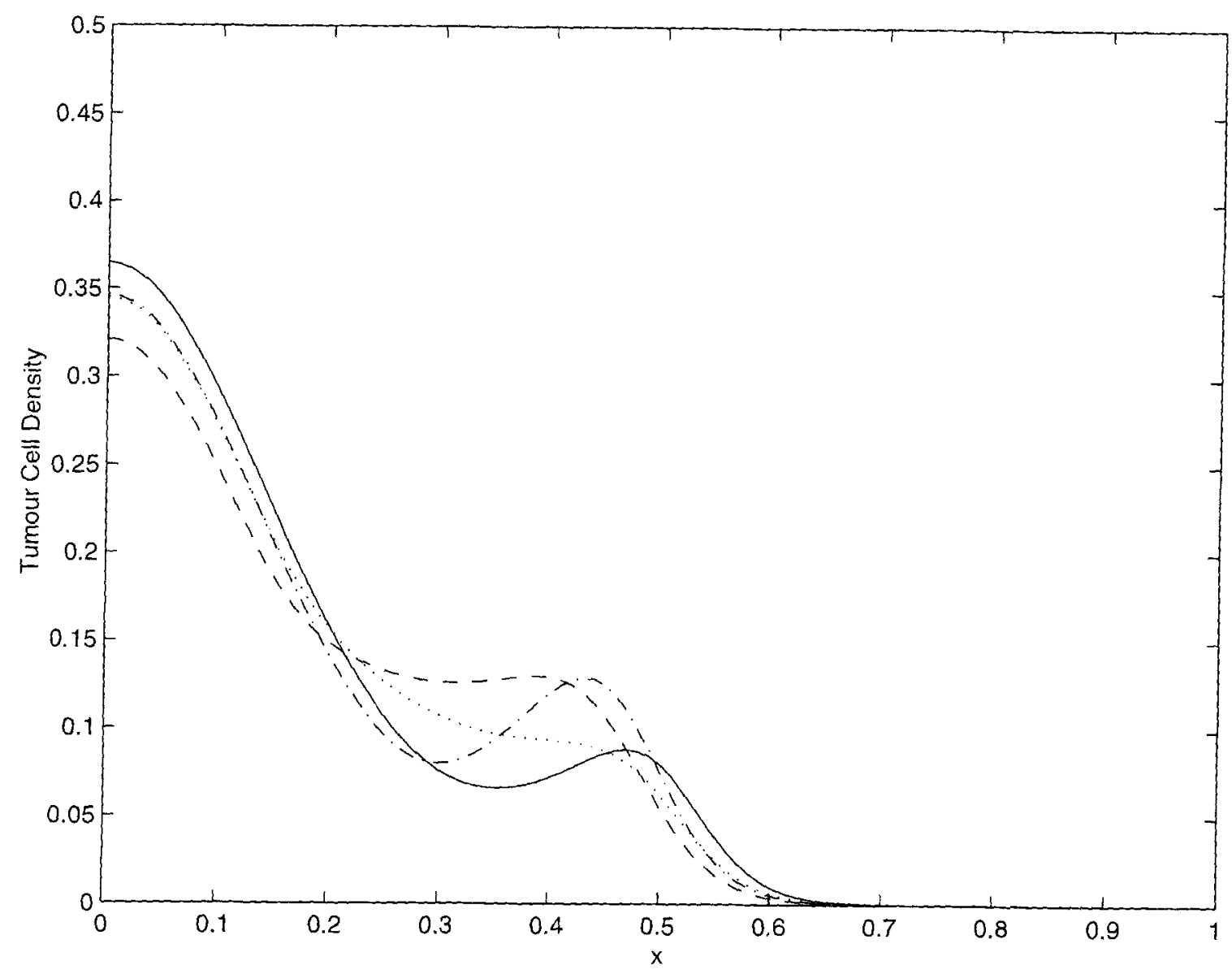

FIGURE 6(a) One dimensional numerical solutions of the system (5), with parameter values as in Figure 1 (unless otherwise stated), showing the (a) cell density, (b) MDE concentration (overleaf) and (c) ECM density (overleaf). (a) shows the tumour cell density at $t=10$ for each of the three functional forms of $h$ i.e. $h=0$ (solid line), $h=\beta m$ (dashed line), $h=\beta m f$ (dashed-dotted line) and $h=\beta m(1-f$ ) (dotted line). (b) shows the MDE concentration for each of the three functional form of $h$ at $t=10$, key as in (a). (c) shows the ECM density for each of the three functional form of $h$ at $t=10$, key as in (a).

(continuous line) and one for each of the above functional forms, (i) dashed, (ii) dashed-dotted and (iii) dotted. Clearly (with the given set of parameter values) the impact on the ECM profile is minimal (Figure 6c). However, the MDE concentration profile curves show that for (ii) very little decay is produced but with both (i) and (iii) the MDE concentration is substantially reduced (Figure 6 b) compared with the original. In contrast to this marked difference in the MDE concentration, the tumour cell density curves all look similar (Figure 6a), although the leading group of cells is not so well defined for (i) and (iii). These results indicate that the precise functional form of $h(m, n, f)$ (with the given set of parameters) is not as important as the actual presence of MDE to degrade the ECM i.e. provided there is some net production of MDE the dynamics of the tumour cell density will remain largely unchanged.

The importance of haptotaxis as a mechanism of invasion is obvious from these results. This in turn emphasises the importance of gradients which appear in the degraded ECM. Since the ECM is unlikely to be a constant homogeneous mass, in order to make the model more realistic we must consider a spatially heterogeneous ECM. We examine how this affects the turnour cell density distribution by considering such a heterogeneous ECM in two dimensions in the following section. 


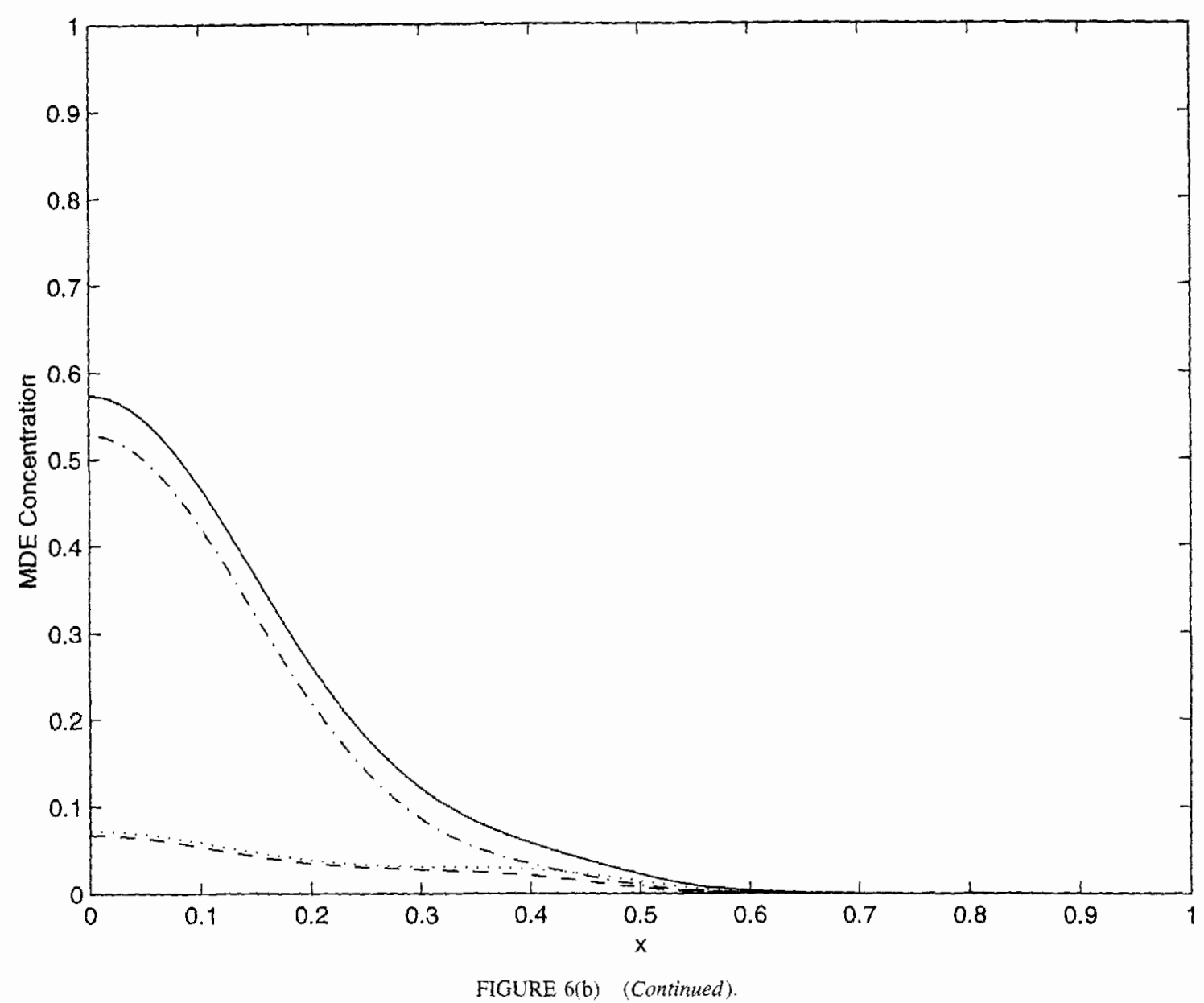

\subsection{Two Dimensional Numerical Simulations}

The aim of this section is to extend the model to a two dimensional spatial domain and therefore to allow the spatio-temporal dynamics of the model to be explored in more detail. All of the numerical solutions presented in this section were obtained from a finite difference approximation of the system (5) with boundary and initial conditions (6)-(9). Since there are no birth and death terms in the tumour cell equation (5) and we impose zero flux boundary conditions (6) then the total cell number is conserved. We used the conservation of cell number as a check on the accuracy of our numerical scheme which was found to be accurate to within $0.01 \%$. The parameter values used in the following simulations (unless specified otherwise) were the same as those used in the one dimensional simulations of Figure 1 i.e. $d_{n}=0.001, d_{m}=0.001, \gamma=0.005, \eta=10, \alpha=$ $0.1, \beta=0$ and $\varepsilon=0.0025$

We first of all consider a homogeneous ECM in two dimensions, thereby extending the one-dimensional results of the previous section. This will also permit us to compare the effect of a heterogeneous ECM in subsequent simulations. Thus, initially we assume that we have a circular initial tumour cell distribution given by (9) and an ECM distribution given by $f(x, y, 0)=1-0.5 n(x, y, 0)$. Finally, we assume that the initial MDE concentration profile is proportional to the initial tumour cell density and take $m(x, y, 0)=$ $0.5 n(x, y, 0)$. The tumour cell initial conditions are illustrated graphically at $t=0$ in Figure 7 .

Figure 7 shows the results of a numerical simulation of equation (5) in $2 \mathrm{D}$. The figure shows 


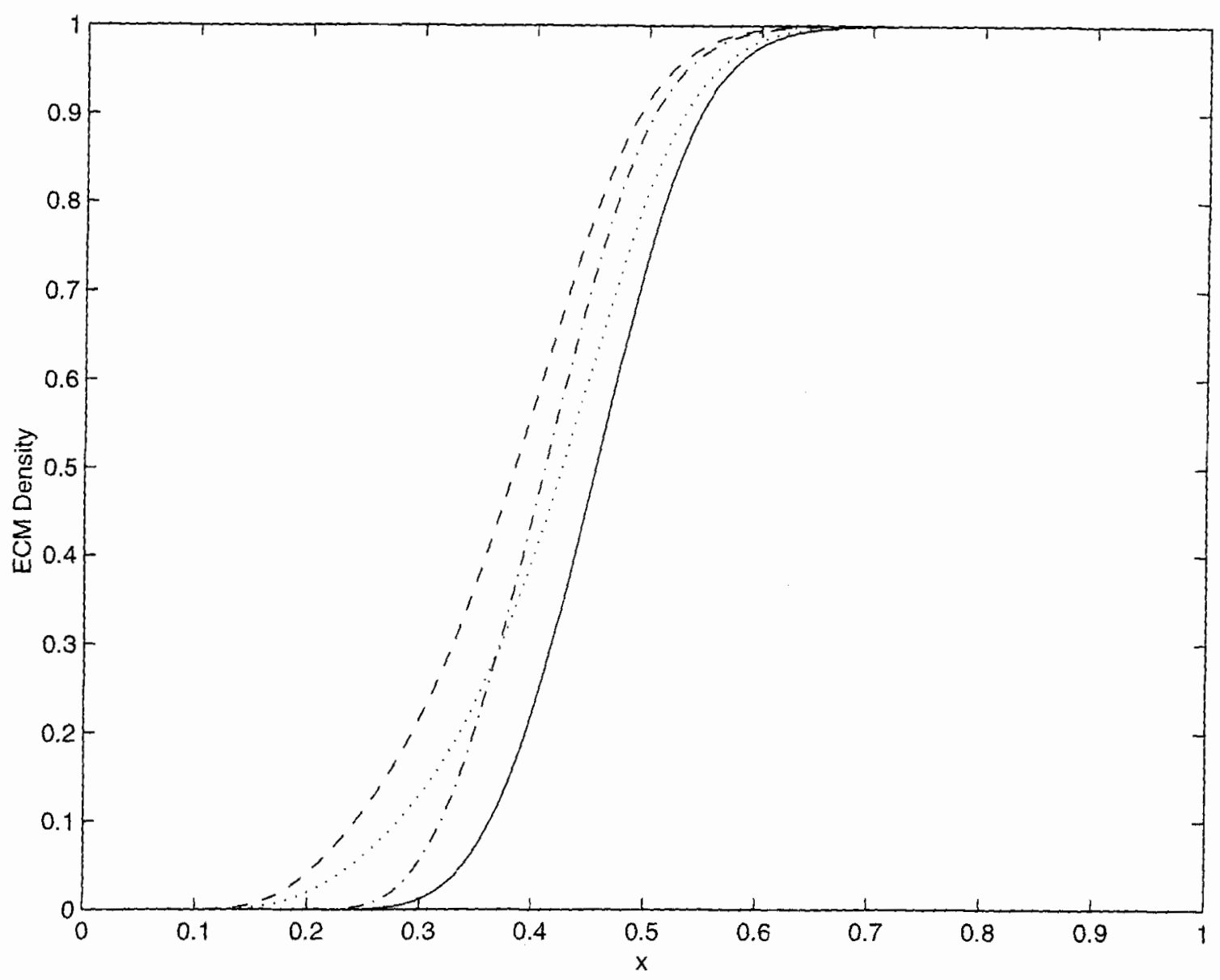

FIGURE 6(c) (Continued).

four snapshots in time of the tumour cell density distribution, with the first figure representing the initial data, as described above. As expected, we see the main body of the tumour invading slowly. At the leading edge there is a region of higher density of cells, which eventually breaks away as a separate ring of cells and invades further into the ECM. These results are as expected, and are in keeping with the analogous one-dimensional results of the previous Section 3.1.

To examine the importance of the role of ECM in the invasive process, we now consider a hypothetical heterogeneous ECM with an initial distribution given in Figure 8 i.e. there are now regions of high density of ECM and regions of low density of ECM. Using this initial ECM data, the same initial tumour cell distribution and MDE concentration as for Figure 7 and the same parameter values, we obtained the results shown in Figures 9 and 10. From Figure 9 we note that the same behaviour is observed at the early stages $(t=1.0,2.0)$ as for Figure 7 with a basic radial expansion. However, by $t=4.0$ the perfect symmetry of the initial tumour cell distribution is broken and there are several regions of higher tumour cell density. The distinction between cells which are mainly driven by diffusion and those driven by haptotaxis is no longer obvious. At later times, from Figure 10, we see that two regions of high cell density form $(t=7.0)$ and continue to invade $(t=10.0)$. The main body of the tumour is approximately bounded by these higher density regions, although by $t=12.0$ the higher density regions have fragmented and a new 'hotspot' has 

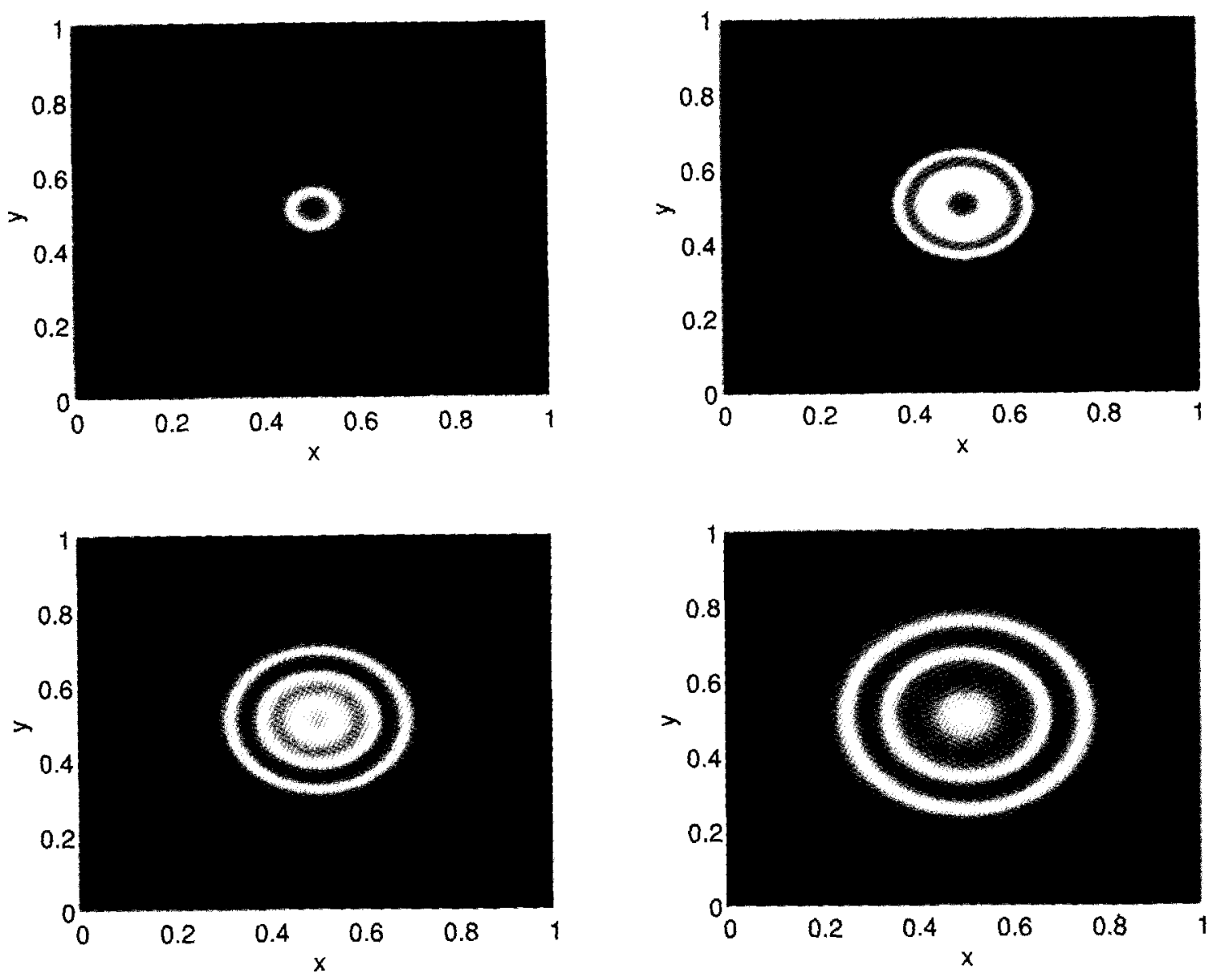

FIGURE 7 Spatio-temporal evolution of the tumour cell density from a numerical simulation of system (5) with constant tumour cell diffusion, representing tumour invasion (see text for parameter values). The figure shows that a ring of cells break from the main body of the tumour and invade further into the ECM. Colour graduation is directly proportional to cell density i.e. red is high density and dark blue low density (see colour plate 1).

appeared. The final figure at $t=15$ shows that the tumour cell density has spread through most of the domain in a somewhat heterogeneous manner with a couple of 'hotspots'. This form of tumour cell density distribution is closer to what is observed in real life than those of Figure 7 and further emphasises the importance of tumour cell/ECM interactions (cf. Figures 14, 15 in the discussion section).

The particular choice of initial ECM distribution (Figure 8) is perhaps somewhat exaggerated and was selected to emphasise the importance of ECM gradients. However, other forms of initial ECM distribution would produce qualitatively similar final results i.e. a heterogeneous tumour cell density distribution.
The two important factors governing the final tumour cell density distribution are ECM heterogeneity and the haptotactic response of the cells to the gradients created in the degraded matrix. These results are in qualitative agreement with actual clinical observations i.e. it is well-known that small clusters of cells can break away from the central mass of the tumour and invade further leading to possible metastasis (cf. Figures 14,15 in the final section).

Whilst the results of Sections 3.1, 3.2 give an indication of the macroscopic behaviour of our model and produce qualitatively realistic results, they are limited in their quantitative capacity and do not account for other important processes such as cell 


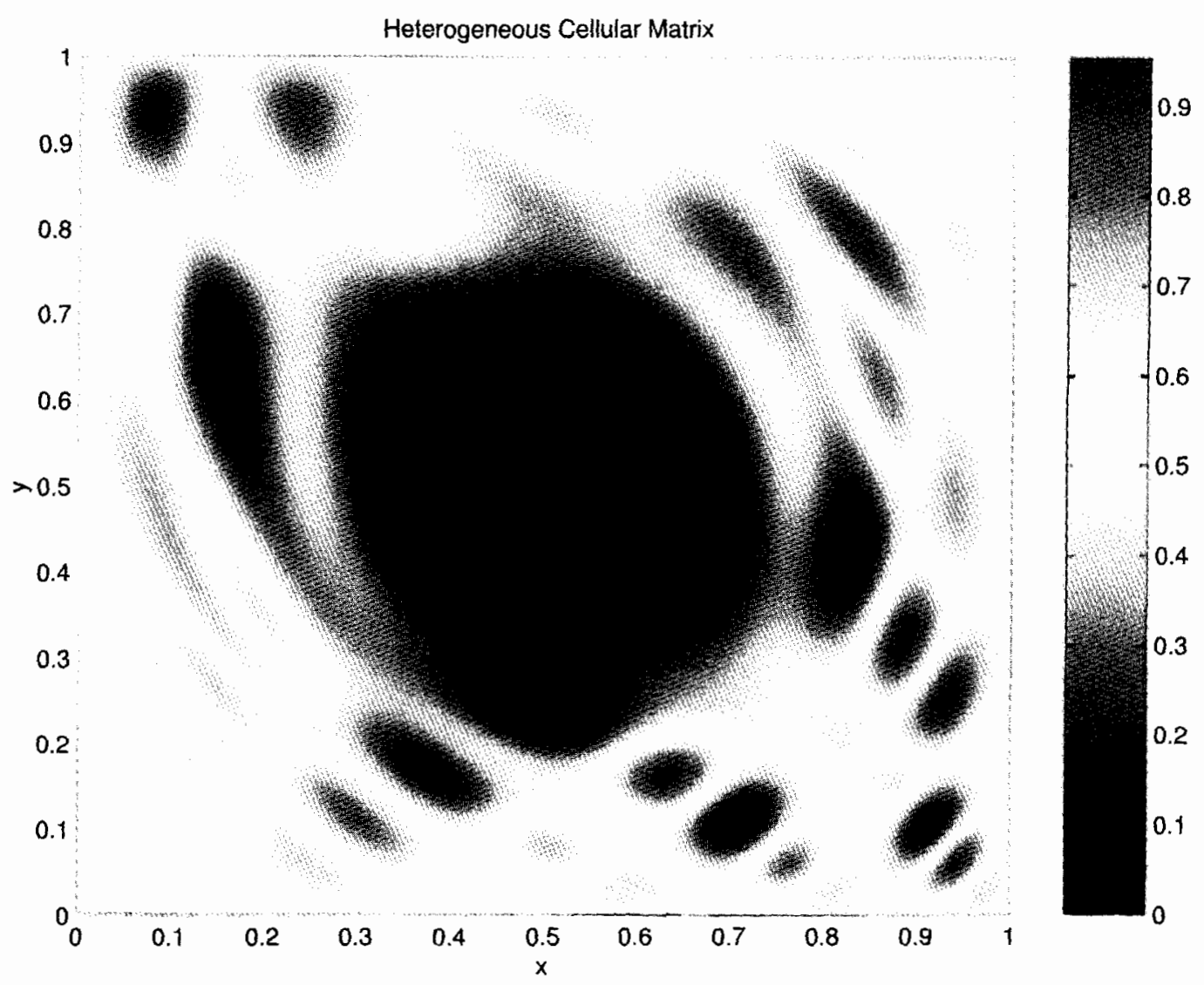

FIGURE 8 Pictorial representation of a hypothetical heterogeneous ECM. Colour graduation is directly proportional to ECM concentration i.e. red is high density and dark blue low density (see colour plate II).

proliferation, cell mutation and individual cell-cell interactions. In the following section we present a discrete model that has the capacity to include all of these processes in a realistic manner and produce both spatial and temporal data on individual invading cells.

\section{THE DISCRETE MATHEMATICAL MODEL}

Discrete mathematical models of tumour invasion already exist in the research literature, but these mainly involve the use of cellular automata. For example, the work of Smolle and Stettner (1993), Smolle and Grimstad (1992). Smolle et al. (1990) concerns invasive patterns generated from a cellular automaton which are compared statistically with experimental results in order to detect real invasive patterns. The model allows for an estimation of cell motility and proliferation. Qi et al. (1992) developed a cellular automaton model of cancerous growth which was compared with experimental growth curves and shown to agree well. Both of these models included cell proliferation and migration terms. Qi et al. (1992) also included the mechanical pressure within the tumour and Smolle and Stettner (1993) consider a further level of complexity with the influence of autocrine and paracrine chemicals.

In this section we will develop a discrete mathematical model of tumour invasion which will enable not only a qualitative but also a quantitative comparison with in vivo experimental results. The particular technique which we will use to follow the path of an individual tumour cell is an implementation of the method developed by Anderson et al. (1997) 

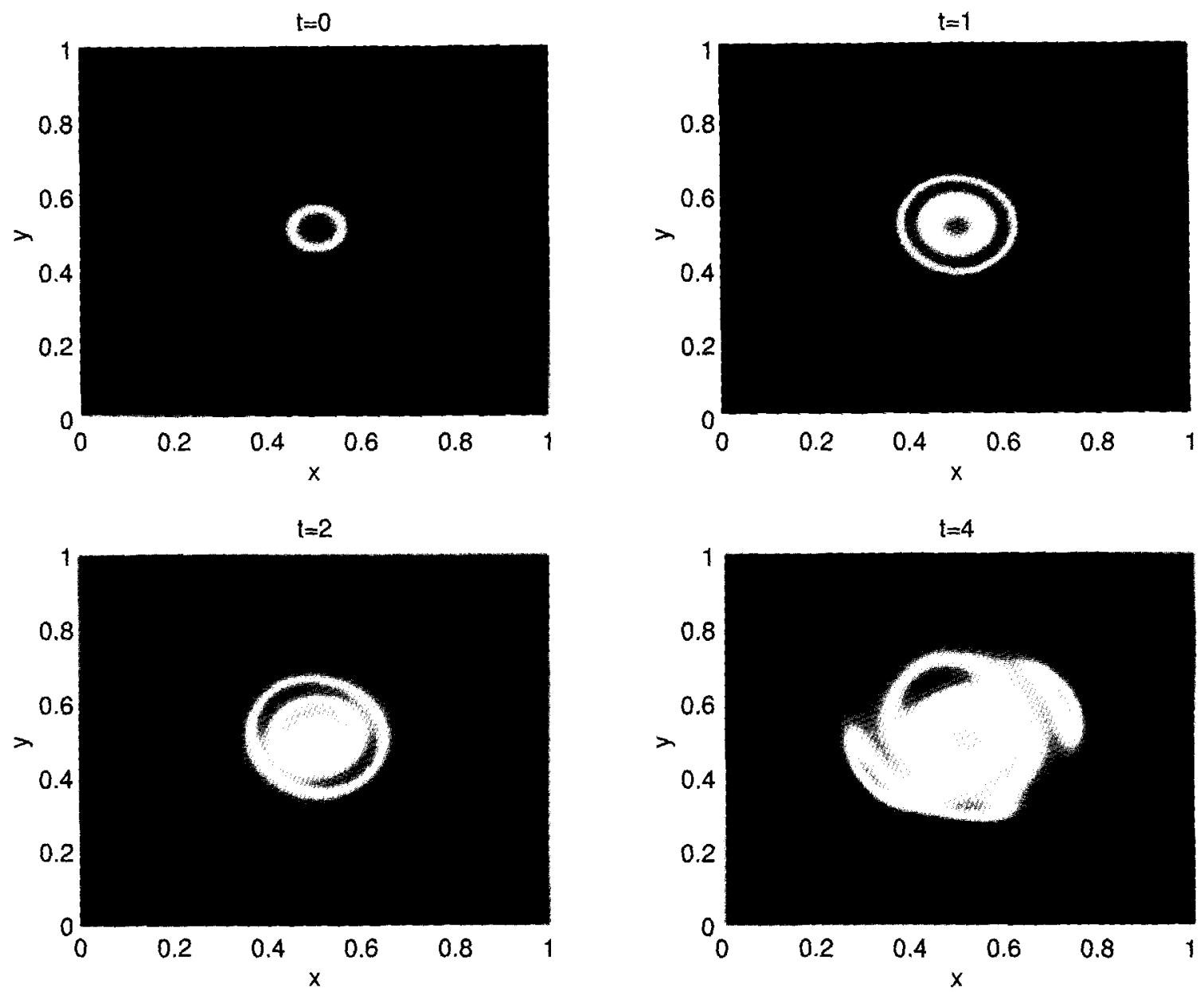

FIGURE 9 Spatio-temporal evolution of the tumour cell density from a numerical simulation of system (5) within a heterogeneous ECM (see text for parameter values). The effect of the ECM on the tumour cell density only becomes apparent for the later values of $t$, where the ring of cells is seen to no longer exist. Colour graduation as in Figure 7 (see colour plate III).

and Anderson and Chaplain (1998) and first of all involves discretizing (using standard finite-difference methods) the partial differential equation governing the rate of change of tumour cell density (5). We then use the resulting coefficients of the five-point finite-difference stencil to generate the probabilities of movement of an individual cell in response to its local milieu. This technique differs from previous discrete models such as Smolle and Stettner (1993) and Qi et al. (1993) in that the movement of individual cells is based on a discrete form of the continuous model. However, like both of these models there is an element of stochasticity (randomness) in the movement rules for the cells. In effect, we will derive a biased random walk governing the motion of a single tumour cell based on the system of partial differential equations (5) of Section 2. In this sense, our discrete model is most similar in formulation to the reinforced random walk models of Othmer and Stevens (1997), where cell movement is modelled in response to a chemical stimulus by considering an equation (discrete in space and continuous in time) governing the probability that a cell is at a given position at time $t$. This equation is a function of the transition probabilities for one-step jumps to the orthogonal neighbours. The form of the transition 

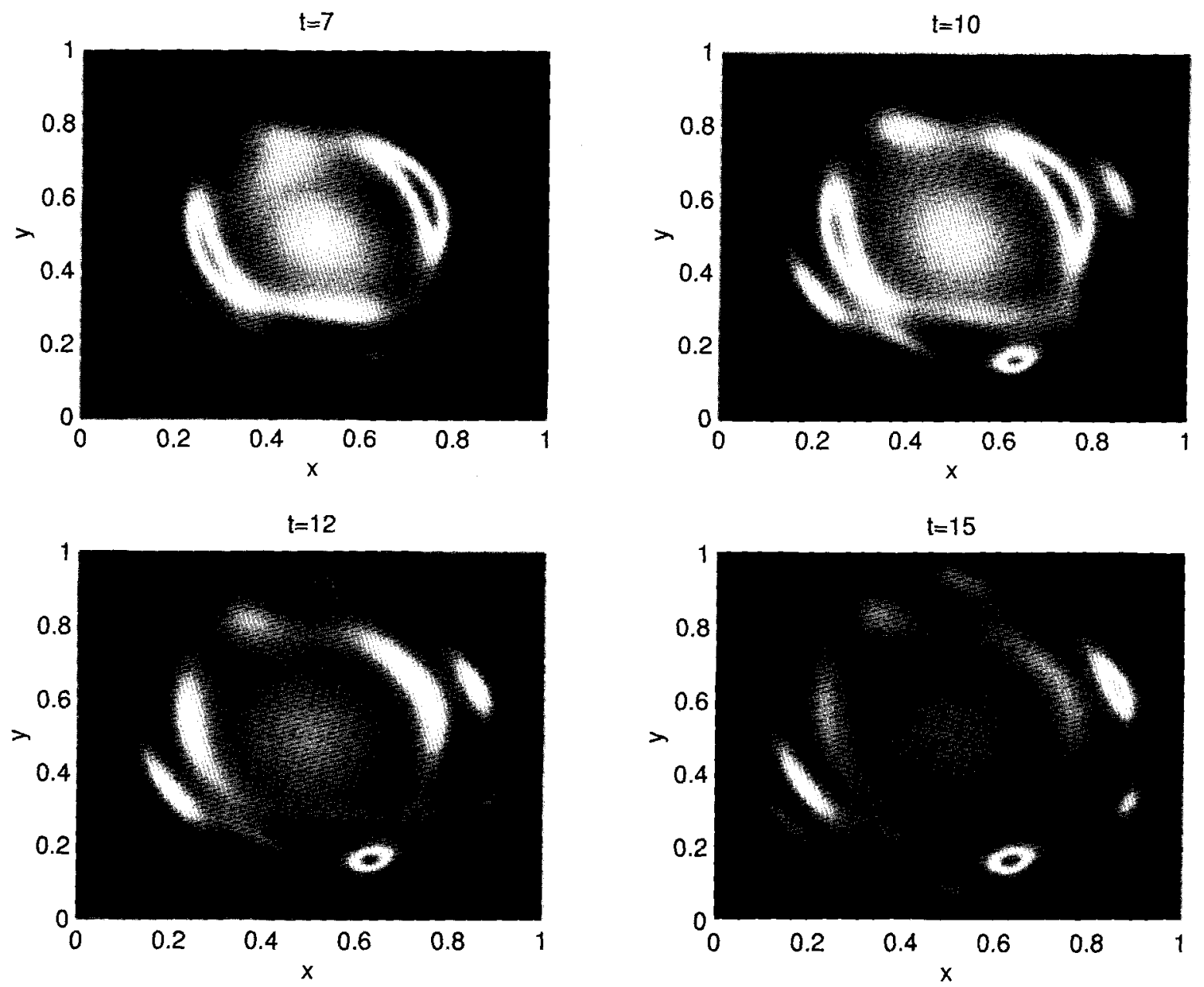

FIGURE 10 Spatio-temporal evolution of the tumour cell density from a numerical simulation of system (5) within a heterogeneous ECM, for later values of $t$ (see text for parameter values). The effect of the heterogeneous ECM via haptotaxis on the tumour cells is now apparent, with the cells invading the ECM in a more heterogeneous manner - resulting in the appearance of 'hot spots'. Colour graduation as in Figure 7 (see colour plate IV).

probabilities for the gradient model of Othmer and Stevens (1997) is very similar to the probabilities of movement that will be derived from our discrete model (see also Alt, 1980; Davis, 1990).

We now set about formulating the discrete model and deriving the movement probabilities for an individual tumour cell in response to its surrounding matrix. The implementation of the process of cell proliferation will be described later. We first discretize (5) using the Euler finite difference approximation (Mitchell and Griffiths, 1980). This involves approximating the continuous two dimensional domain $[0,1] \times[0,1]$ in the usual way as a grid of discrete points (mesh size $h$ ), and time $(t)$ by discrete increments (magnitude $k$ ). The full discretized system is given in the Appendix. For clarity we only consider the tumour cell equation,

$$
\begin{aligned}
n_{i, j}^{q+1}= & n_{i, j}^{q} P_{0}+n_{i+1, j}^{q} P_{1}+n_{i-1, j}^{q} P_{2} \\
& +n_{i, j+1}^{q} P_{3}+n_{i, j-1}^{q} P_{4}
\end{aligned}
$$

where the subscripts specify the location on the grid and the superscripts the time steps. 
That is $x=i h, y=j h$ and $t=q k$ where $i, j, k, q$ and $h$ are positive parameters.

In a numerical simulation of the continuous model (5), the purpose of the discrete equation (10) is to determine the tumour cell density at grid position $(i, j)$, and time $q+1$, by averaging the density of the four surrounding neighbours at the previous time q. For our discrete model, we will use the five coefficients $P_{0}$ to $P_{4}$ from (10) to generate the motion of an individual tumour cell. These coefficients can be thought of as being proportional to the probabilities of the tumour cell being stationary $\left(P_{0}\right)$ or moving left $\left(P_{1}\right)$, right $\left(P_{2}\right)$, up $\left(P_{3}\right)$ or down $\left(P_{4}\right)$.

Each of the coefficients $P_{1}$ to $P_{4}$ consist of two components,

$$
P_{n}=\text { Random movement }+ \text { Haptotaxis }
$$

thus showing how the discrete tumour cell equation is linked to the continuous tumour cell equation of system (5). The coefficient $P_{0}$ has a similar form (see Appendix). Equation (11) is very similar to the transition probabilities of the reinforced random walk model of Othmer and Stevens (1997). In particular, their gradient models have a random component and a "taxis" component. Othmer and Stevens (1997) used their discrete transition probabilities to then derive a partial differential equation in the continuous limit. It is possible to show this for our model by defining transition probabilities of the form (11). The original equation governing the rate of change of tumour cell density (5) can then be recovered by following the analysis of Othmer and Stevens (1997) in the same rigorous manner.

The exact forms of $P_{0}$ to $P_{4}$ are functions of the ECM density near an individual tumour cell (see Appendix). Therefore, if there were no ECM the values of $P_{1}$ to $P_{4}$ would be equal, with $P_{0}$ smaller (or larger, depending on the precise values chosen for the space and time steps) i.e. there is no bias in any one direction and the tumour cell is less (more) likely to be stationary - approximating an unbiased random walk. However, if there are gradients in the ECM, haptotaxis dominates and the coefficients $P_{0}$ to $P_{4}$ will become biased towards the direction of increased ECM density. The motion of an individual cell is therefore governed by its interactions with the matrix macromolecules in its local environment.

Before proceeding to the simulation section, we first of all discuss the manner in which we explicitly incorporate cell proliferation into the discrete model.

\section{Cell Proliferation}

In our model we assume that each individual cell has the capacity for proliferation and will produce two daughter cells provided the following two conditions are satisfied: (i) the parent cell has reached maturity and (ii) there is sufficient space surrounding the parent cell for the two new daughter cells to move into. We defined cell maturity to be 500 discrete time steps. While this timescale is arbitrary, with a precise estimate of parameter values in the original model, this maturity time can be made to correspond with an actual cell cycle time for specific cancer cells. In order to satisfy condition (ii), we assumed that a daughter cell could arise if any one of the parent cell's four orthogonal neighbours was empty. If more than one of the neighbouring grid points is empty then the new cell position is chosen randomly from these points. In order to keep the running time of simulations within reasonable limits we have restricted the maximum number of cells to 3000 , with an initial distribution of 500 cells.

\section{Simulation Process for the Discrete Model}

Each time step of the simulation process involves solving the discrete form of the system (5) numerically to generate the five coefficients $P_{0}$ to $P_{4}$ (see Appendix). Probability ranges are then computed by summing the coefficients to produce 5 ranges, $R_{0}=0$ to $P_{0}$ and $R_{m}=\sum_{l=0}^{m-1} P_{l}$ to $\sum_{l=0}^{m} P_{l}$, where $m=1$ to 4. We then generate a random number between 0 and 1 , and depending on the range which this number falls in, the current individual tumour cell under consideration will remain stationary $\left(R_{0}\right)$ or move left $\left(R_{1}\right)$, right $\left(R_{2}\right)$, up $\left(R_{3}\right)$ or down $\left(R_{4}\right)$. The larger a particular range, the greater the probability that the corresponding coefficient will be selected. Each 
tumour cell is therefore restricted to move to one of its four orthogonal neighbouring grid points or remain stationary at each time step.

All the simulations of the discrete model were carried out on a $200 \times 200$ grid, which is a discretization of a the unit square, $[0,1] \times[0,1]$, with a space step of $h=0.005$ and a time step of $k=0.001$. A discrete form of the no flux boundary condition (6) was imposed on the square grid, restricting the tumour cells to within the grid. The initial conditions in all simulations (unless otherwise stated) are given by discrete forms of (7) and (9) with an initial number of 500 tumour cells centred around $(0.5,0.5)$.
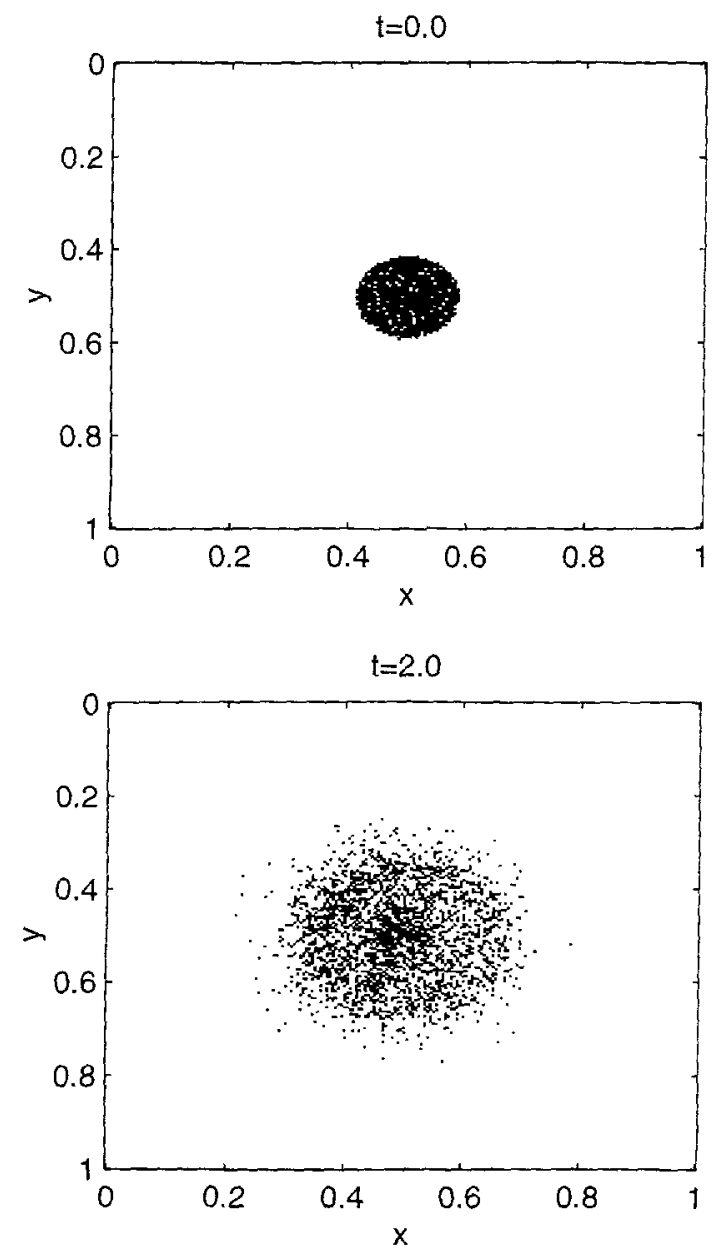

The parameter values used in the following simulations are the same as those used in the previous two dimensional continuous simulations (unless otherwise stated) i.e. $d_{n}=0.001, d_{m}=0.001$, $\gamma=0.005, \eta=10, \beta=0$ and $\alpha=0.1$.

\section{DISCRETE MODEL SIMULATION RESULTS}

As with the continuous two dimensional simulations we will initially consider our discrete model with a homogeneous initial ECM density profile. Figure 11 shows four snapshots in time of the tumour cell
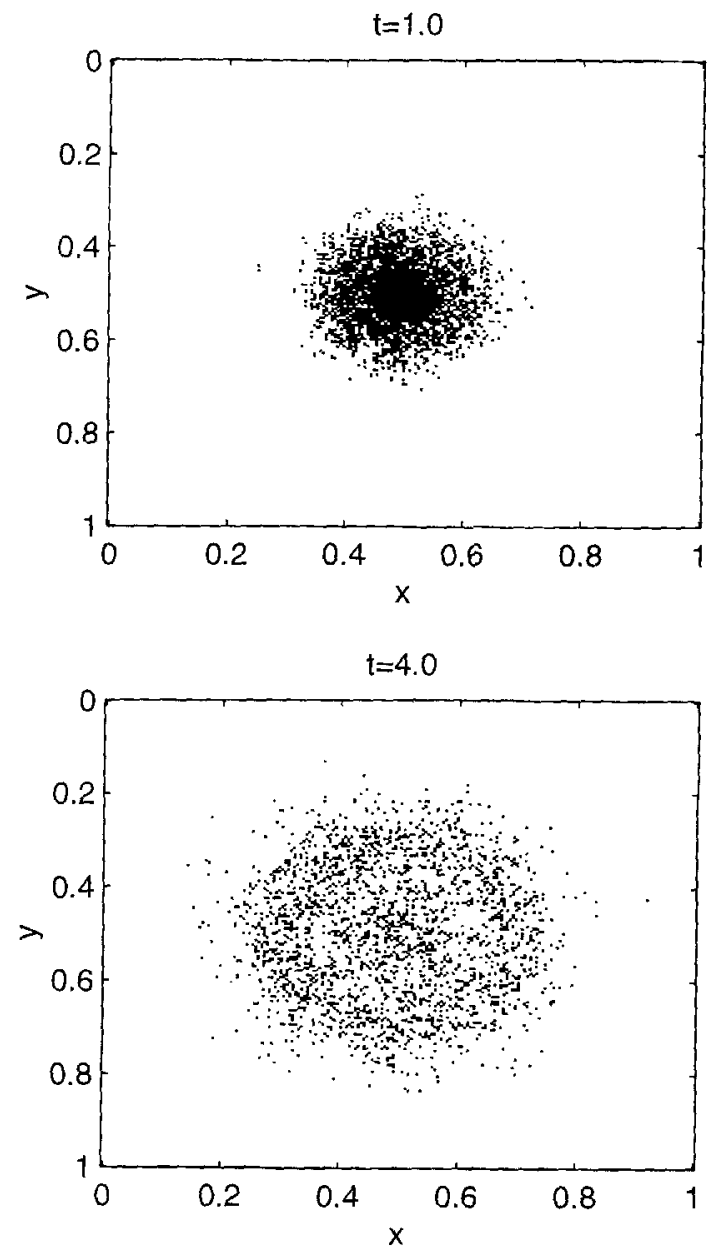

FIGURE 11 Spatio-temporal evolution of tumour cell invasion from a numerical simulation of the discrete model. The figure shows the tumour cells migrating from the centre $(x=0.5, y=0.5)$ into the ECM (see text for parameter values). We observe that the overall distribution of the cells is very similar to the continuous equivalent (Figure 7) and that a few individual cells invade further into the ECM than the main body of the cells. 

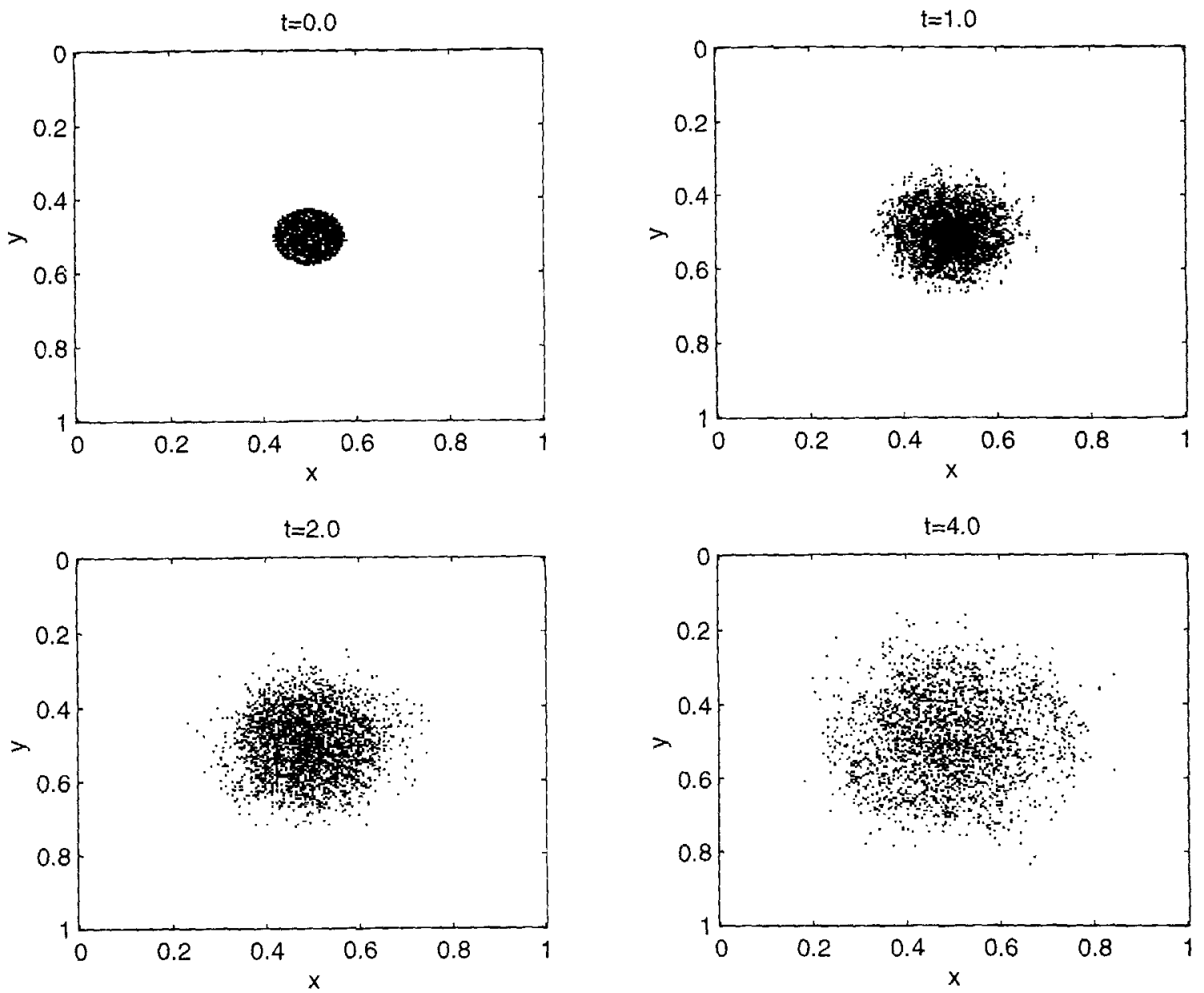

FIGURE 12 Spatio-temporal evolution of tumour cell invasion from a numerical simulation of the discrete model. The figure shows the tumour cells migrating from the centre $(x=0.5, y=0.5)$ into the heterogeneous ECM as given in Figure 8 (see text for parameter values). No real structure is apparent but the cell distribution is clearly different from Figure 11 and again a few individual cells are seen to invade further into the ECM

invasion process. From the initial cluster (shown as $t=0.0$ ) the tumour cells at the leading edge are seen to migrate the most. As time evolves the ring-like structure observed in the continuous results (Figure 7) can be seen $(t=4.0)$. However, the most striking feature of these results is to notice that a few individual tumour cells migrate much further into the ECM separated from the main tumour mass. These cells have the greatest potential to metastasise further and are difficult to detect clinically. It should be emphasised that the movements of the individual cells, whilst governed by the continuous model via the discretisation, do have a genuine stochastic component and the cell movements can therefore deviate from the continuous results. Also since the discrete model incorporates cell proliferation, whereas the continuous model does not, we expect to see some differences. However, the total cell number is limited to a maximum of 3000 cells and therefore the structures seen in Figure 11 are produced mainly by cell migration i.e. random motility and haptotaxis, rather than cell proliferation.

We now examine tumour cell invasion in a heterogeneous ECM. Using a discrete form of Figure 8 for the initial ECM concentration and the same parameters as above, we obtained Figures 12 and 13. From the initial cluster (at $t=0.0$ ) cells begin to migrate in a very similar manner to those observed in Figure 11. 

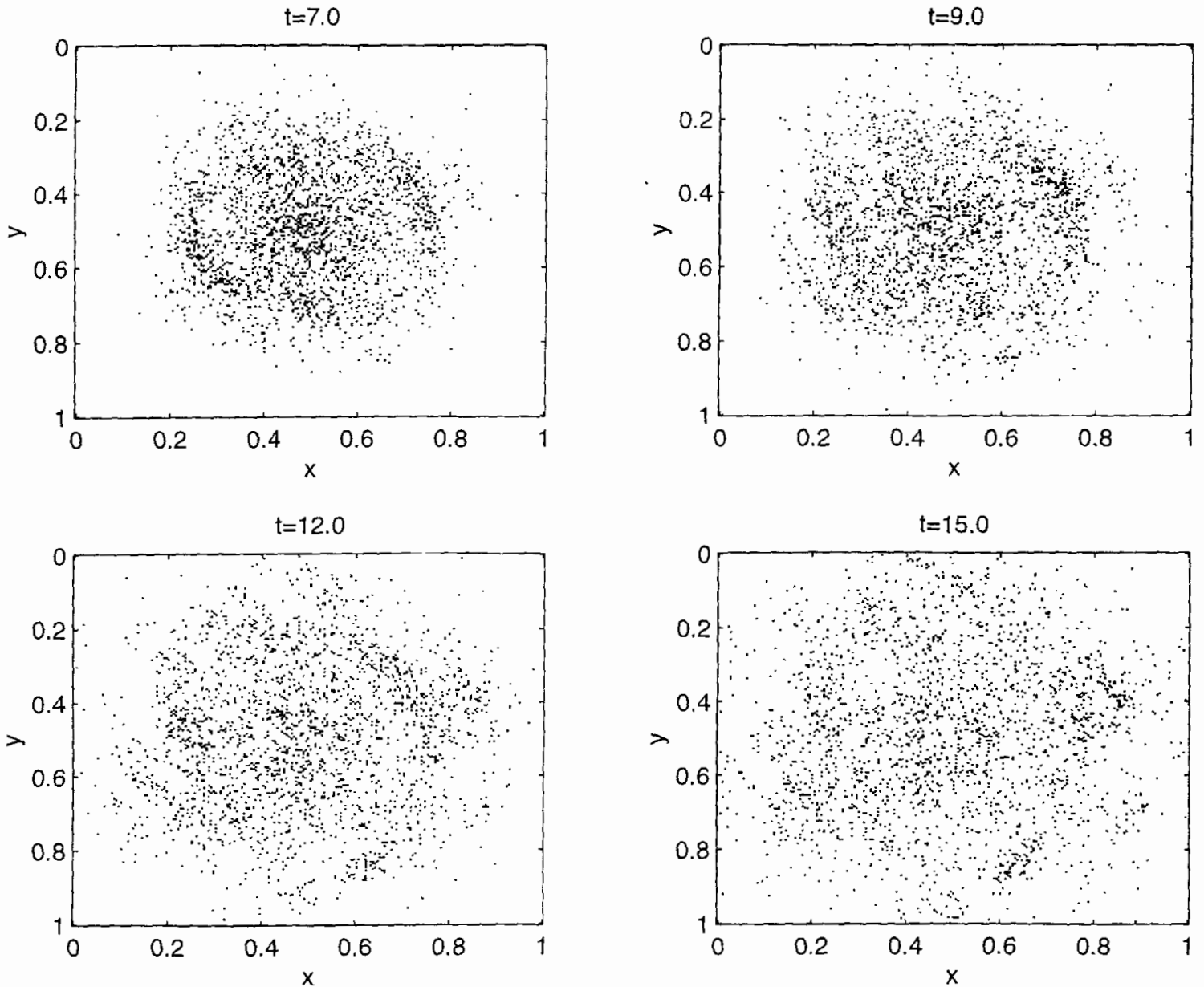

FIGURE 13 Spatio-temporal evolution of tumour cell invasion from a numerical simulation of the discrete model. The figure shows the tumour cells migrating into the heterogeneous ECM as in Figure 8 but for later values of $t$ (see text for parameter values). We now observe that the overall distribution of the cells is very similar to the continuous equivalent (Figure 10) and a few individual cells have in fact reached the boundaries of the domain.

However, by $t=2.0$ the ring-like clustering of the cells is not seen and this is further emphasised at $t=4.0$. Again we see individual cells migrating out further than the main group. The patterning observed in the comparable continuous results (Figure 9) is not as obvious, but as time evolves we can see from Figure 13 (at $t=7.0$ ) the two regions of increased cell density that are equivalent to the two regions of higher density seen in Figure 10 at $t=7.0$. As $t$ increases the cells migrate further into the ECM and become more dispersed, although, small clusters can still be observed e.g. just below $x=0.6, y=0.8$ for $t=12.0-15.0$. This again is in agreement with the continuous results (Figure 10). By $t=15$ quite a few of the cells have already reached the boundary of the domain, which is something that did not occur in the continuous model simulations. This further illustrates the importance of the ECM structure in aiding or hindering the migration of individual cells that have the potential to metastasise.

\section{DISCUSSION AND CONCLUSIONS}

The work we have presented here has developed a mathematical model for tumour invasion using a 
novel blend of continuum, deterministic modelling and discrete, stochastic modelling in 1 and 2 space dimensions.

The continuum model consists of a system of nonlinear partial differential equations and examines how tumour cells respond to ECM gradients via haptotaxis, created both by the tumour cells through MDE degradation of the matrix and those already in existence within the matrix. The results from the one dimensional continuum-model simulations demonstrate the impact of interactions between tumour cells and the ECM on possible metastasis. In particular if tumour cells move via random migration and haptotaxis and the intensity of the random movements is dependent upon MDE concentration then a small cluster of cells can easily break away from the main body of the tumour (Figure 2). Even without this MDE dependence, it is clear that the tumour cells can split into two groups: those driven by random migration and those driven by haptotaxis (Figure 1). However, this result of the model is mainly due to the fact that the only gradients in the ECM are a result of MDE degradation and hence the cells at the leading edge of the tumour are mostly affected by haptotaxis. When ECM heterogeneity is introduced, in the two dimensional simulations, this grouping of cells into those driven mainly by random migration and those driven mainly by haptotaxis is no longer obvious because of the gradients already existing within the ECM. The heterogeneous ECM (Figure 8) is more likely to be characteristic of real ECM within the body and the resulting tumour cell density distributions are more realistic (Figures 9-10) i.e. a heterogeneous tumour cell density with a few 'hotspots'. Indeed, in Figure 14, we present a figure of an actual mammogram of a breast cancer. The contrast arises from the deposition of calcium (microcalcification), which is a common finding in this disease. The central tumour mass can clearly be seen, but also some contrast-bright specks around it, which may represent clusters of tumour cells which have already broken away from the central mass.

The discrete model that we developed was derived from a discretized form of the partial differential equations of the continuum model, and permits the tracking of individual tumour cells and also enables us to explicitly incorporate rules for cell proliferation. With reference to the larger scale, the results from the discrete model confirm the predictions of the continuum model that haptotaxis is important for both invasion and metastasis. On a finer scale, the discrete results show that cell proliferation can aid in invasion as a result of space filling. Also, the ECM structure (via haptotaxis) may aid individual cells in breaking from the main body of the tumour and thus escaping to become possible metastases (Figures 12-13). The discrete results were also able to show that many cells invade further into the ECM than is predicted from the continuous results - which again has important implications for metastasis.

To some extent the discrete model is still under development and it has the potential to include more processes than just cell proliferation. For example specific cell-cell interactions could be modelled, such as contact inhibition or cell-cell adhesion. Genetic information about each cell can be stored and passed from generation to generation incorporating the possibility of genetic mutations. These may then alter the cell proliferation rate, migration rate, adhesion properties, or apoptotic rate. If exact parameter values were obtained for the discrete model then it would be possible to obtain the physical cell numbers that are falling within a given radius of the main tumour mass and could therefore be used as a predictive tool for estimating how far a surgeon should cut to ensure all of the tumour is removed. To emphasise this point and to show that our model reproduces clinically observed invasion patterns, we present the results of the histological section of a breast cancer, stained with haematoxylin and eosin, in Figure 15. The tumour tissue is to the top and right, with the normal tissue to the bottom and left. Clearly visible is the small group (or "nest") of tumour cells, well in advance of the invasive front.

The technique of using partial differential equations as the basis for discrete models is clearly very useful, with the ability to generate movements of individual cells based on a continuum model of a population of cells. Indeed, this technique provides a powerful means of linking micro-scale events to macro-scale 


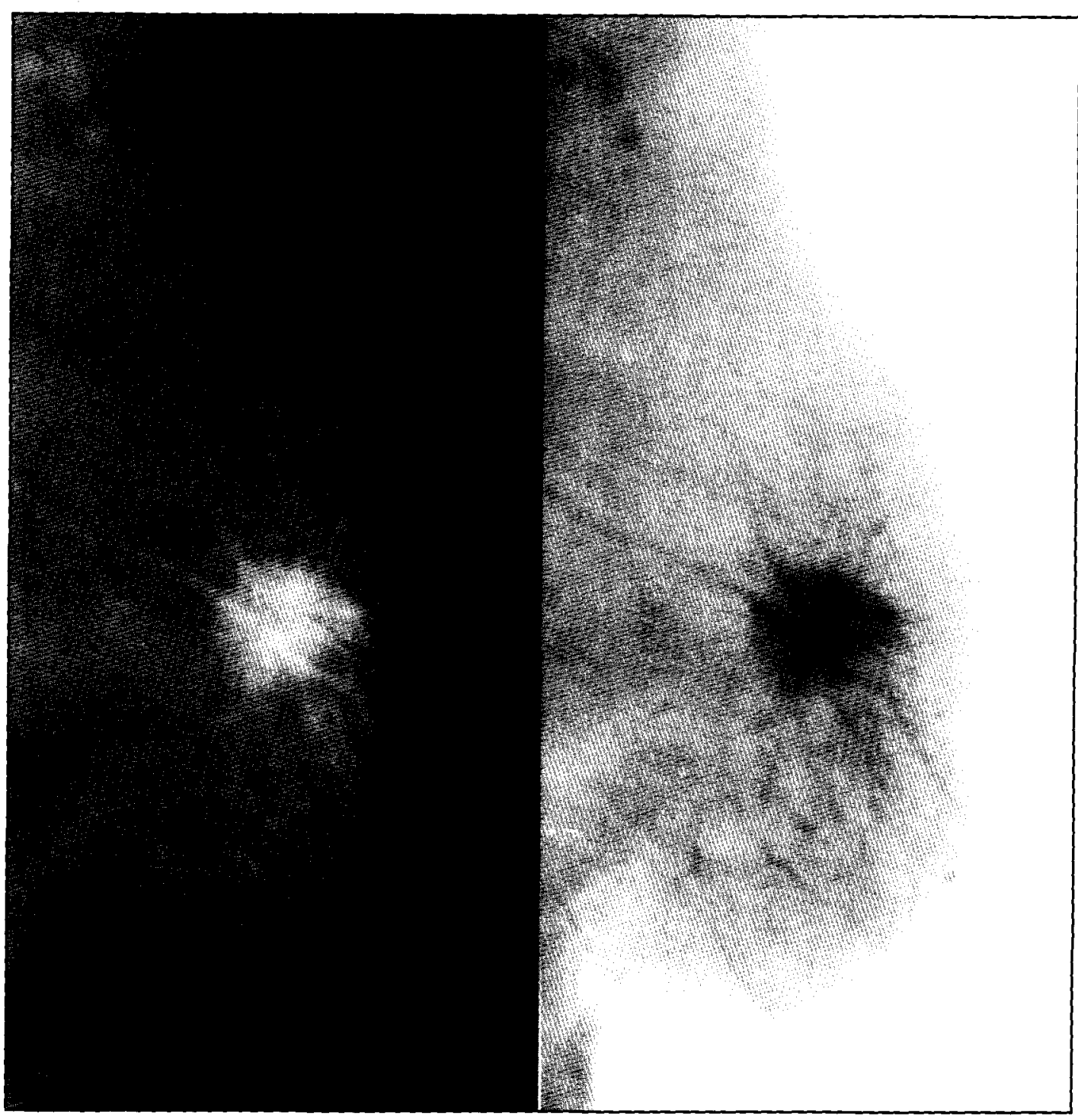

FIGURE 14 Mammogram of a breast cancer. The contrast arises from the deposition of calcium (microcalcification), which is a common finding in this disease. Note the central tumour mass, but also some contrast-bright specks around it, which may represent clusters of tumour cells.

events, individual behaviour to population behaviour, with potential application to a wide range of problems in mathematical biology.

From a clinical point of view, these models have enormous potential. Even at this stage, the behaviour of the simulated tumours closely parallels histological observations, especially when a heterogeneous ECM is introduced (cf. Figures 9, 10 with Figure 14; cf. Figures 12, 13 with Figure 15). It is therefore conceivable that measurement in tumours of some of the parameters used in these models will provide precise information on the invasive behaviour of individual neoplasms. For example, it should then be possible to estimate the likely extent of local infiltration by a tumour and thereby tailor the radicality of surgical excision for that individual situation. It may also be possible to assess more accurately than at present the likelihood of metastatic disease, which 


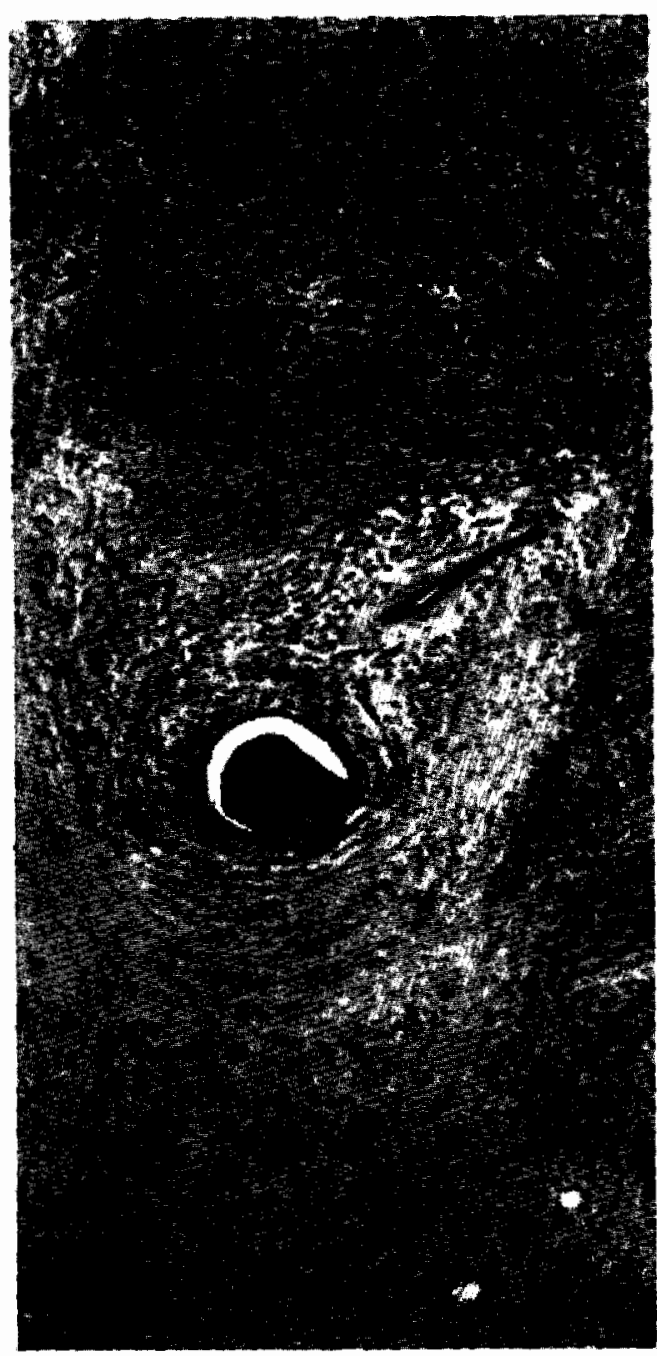

FIGURE 15 Histological section of a breast cancer, stained with haematoxylin and eosin. Tumour tissue is to the top and right, normal tissue to the bottom and left. Note the nest of tumour cells, well in advance of the invasive front (see colour plate $\mathrm{V}$ ).

will have important implications for adjuvant systemic therapy.

\section{World Wide Web}

Results from further numerical simulations of the model (including MPEG animations) can be found at the URL

http : //www.mcs.dundee.ac.uk : 8080/

$\sim$ sanderso/invasion/

\section{Appendix}

To discretize the continuous system (5) we use Euler finite difference approximations (Mitchell and Griffiths, 1980), which leads to the system,

$$
\begin{aligned}
n_{i, j}^{q+1}= & n_{i, j}^{q} P_{0}+n_{i+1, j}^{q} P_{1}+n_{i-1, j}^{q} P_{2}+n_{i, j+1}^{q} P_{3}+n_{i, j-1}^{q} P_{4}, \\
f_{i, j}^{q+1}= & f_{i, j}^{q}\left[1-k \eta n_{i, j}^{q}\right], \\
m_{i, j}^{q+1}= & m_{i, j}^{q}\left[1-\frac{4 k D_{m}}{h^{2}}-k \alpha n_{i, j}^{q}\left(1-n_{i, j}^{q}\right)\right] \\
& +\frac{k D_{m}}{h^{2}}\left[m_{i+1, j}^{q}+m_{i-1, j}^{q}+m_{i, j+1}^{q}+m_{i, j-1}^{q}\right],
\end{aligned}
$$

with $x=i h, y=j h$ and $t=p k$.

The coefficient $P_{0}$, which is proportional to the probability of no movement, has the form,

$$
\begin{aligned}
P_{0}= & 1-\frac{4 k D_{n}}{h^{2}}-\frac{k \gamma}{h^{2}} \\
& \times\left(f_{i+1, j}^{q}+f_{i-1, j}^{q}-4 f_{i, j}^{q}+f_{i, j+1}^{q}+f_{i, j-1}^{q}\right),
\end{aligned}
$$

and the coefficients $P_{1}, P_{2}, P_{3}$ and $P_{4}$, which are proportional to the probabilities of moving left, right, up and down respectively, have the forms,

$$
\begin{aligned}
& P_{1}=\frac{k D}{h^{2}}-\frac{k \gamma}{4 h^{2}}\left[f_{i+1, j}^{q}-f_{i-1, j}^{q}\right], \\
& P_{2}=\frac{k D}{h^{2}}+\frac{k \gamma}{4 h^{2}}\left[f_{i+1, j}^{q}-f_{i-1, j}^{q}\right], \\
& P_{3}=\frac{k D}{h^{2}}-\frac{k \gamma}{4 h^{2}}\left[f_{i, j+1}^{q}-f_{i, j-1}^{q}\right], \\
& P_{4}=\frac{k D}{h^{2}}+\frac{k \gamma}{4 h^{2}}\left[f_{i, j+1}^{q}-f_{i, j-1}^{q}\right] .
\end{aligned}
$$

When there is no ECM concentration in the same region as a tumour cell, $P_{1}$ to $P_{4}$ are equal since the values of $f$ are 0 . Also when there is an equal amount of ECM on either side of a tumour cell (i.e. no gradient), the values $f_{i, j-1}$ and $f_{i, j+1}$ cancel each other out as do $f_{i-1 . j}$ and $f_{i+1, j}$ and thus $P_{1}$ to $P_{4}$ are equal. Therefore, in both these circumstances unbiased random movements will be produced. However, if there is more ECM on one side of the tumour cell than the other, the probabilities ( $P_{1}$ to $P_{4}$ ) will no longer be equal and hence directed movement, towards the higher concentration of ECM, will result. 


\section{Acknowledgement}

This work was supported by BBSRC Grant MMI09008.

\section{References}

Ahmad, A., Hanby, A., Dublin, E., Poulsom, R., Smith, P. Barnes, D., Rubens, R., Anglard, P. and Hart, I. (1998). Stromelysin 3: an independent prognostic factor for relapsefree survival in node-positive breast cancer and demonstration of novel breast carcinoma cell expression. Am. J. Pathol, 152 $721-728$.

Alt, W. (1980). Biased random walk models for chemotaxis and related diffusion approximations. J. Math. Biol, 9, 147-177.

Anderson, A. R. A. and Chaplain, M. A. J. (1998). Continuous and Discrete Mathematical Models of Tumour-Induced Angiogenesis Angiogenesis. Bull. Math. Biol, 60, 857-899.

Anderson, A. R. A., Sleeman, B. D., Young, I. M. and Griffiths, B. S. (1997). Nematode movement along a chemical gradient in a structurally heterogeneous environment II, Theory. Fundam. appl. Nematol., 20, 165-172.

Bafetti, L. M., Young, T. N., Itoh, Y. and Stack, M. S. (1998). Intact vitronectin induces matrix metalloproteinases-2 and tissue inhibitor of metalloproteinases-2 expression and enhanced cellular invasion by melanoma cells. J. Biol. Chem, 273, 143-149.

Beattie, G. J. and Smyth, J. F. (1998). Phase I study of intraperitoneal metalloproteinases inhibitor BB94 in patients with malignant ascites. Clin. Cancer Res, 4, 1899-1902

Bray, D. (1992). Cell Movements, New York: Garland Publishing.

Brown, P. D. (1998). Matrix metalloproteinases in gastrointestinal cancer. Gut, 43, 161-163.

Byrne, H. M., Chaplain, M. A. J., Pettet, G. J. and McElwain, D. L. S. (1999). A mathematical model of trophoblast invasion. J. theor. Med, (in press).

Carter, S. B. (1965). Principles of cell motility: The direction of cell movement and cancer invasion. Nature, 208, 1183-1187.

Chambers, A. F. and Matrisian, L. M. (1997), Changing views of the role of matrix metalloproteinases in metastasis. J. Natl. Cancer Inst., 89, 1260-1270.

Chaplain, M. A. J. and Stuart, A. M. (1993). A model mechanism for the chemotactic response of tumour cells to tumour angiogenesis factor. IMA J. Math. Appl. Med. Biol., 10, 149-168.

Chaplain, M. A. J. and Sleeman, B. D. (1993). Modelling the growth of solid tumours and incorporating a method for their classification using nonlinear elasticity theory. J. Math. Biol, 31, $431-479$.

Chaplain, M. A. J. (1995). The mathematical modelling of tumour angiogenesis and invasion. Acta Biotheor, 43, 387-402.

Chaplain, M. A. J. (1996). Avascular growth, angiogenesis and vascular growth in solid tumours, the mathematical modelling of the stages of tumour development. Mathl. Comput. Modelling, 23, $47-87$.

Davis, B. (1990). Reinforced random walk. Probab. Th. Rel. Fields, 84, $203-229$.

Duichting, W. (1990a). Tumor growth simulation. Comput. and Graphics, 14, 505-508.

Düchting, W. (1990b). Computer simulation in cancer research. In Advanced Simulation in Biomedicine, (D .P. F. Möller, ed), pp. 117-139. Springer-Verlag, New York.

Düchting, W. (1992). Simulation of malignant cell growth. In Fractal geometry and Computer Graphics, (J. L. Encarnção, H.O. Peitgen, G. Sakas, G. Englert eds), pp. 135-143. SpringerVerlag, New York.
Düchting, W., Ulmer, W. and Ginsberg, T. (1996). Cancer, A challenge for control theory and computer modelling. Euro.J. Cancer, 32A, 1283-1292.

Gatenby, R. A. and Gawlinski, E. T. (1996). A reaction-diffusion model of cancer invasion. Cancer Res, , 56, 5745-5753.

Itoh, T., Tanioka, M., Toshida, H., Yoshioka, T., Nishimoto, H. and Itohara, S. (1998). Reduced angiogenesis and tumor progression in gelatinase A-deficient mice. Cancer Res, 58, $1048-1051$.

Kim, J., Yu, W., Kovalski, K. and Ossowski, L. (1998). Requirement for specific proteases in cancer cell intravasation as revealed by a novel semiquantitative PCR-based assay. Cell, 94 $353-362$.

Kimmel, M. and Axelrod, D. E. (1991). Unequal cell division, growth regulation and colony size of mammalian cells, a mathematical model and analysis of experimental data. $J$. Theor. Biol, 153, $157-180$.

Kleiner, D. E. and Stetler-Stevenson, W. G. (1993). Structural biochemistry and activation of matrix metallo-proteases. Curr. Opin. Cell Biol, 5, 891-897.

Klominek, J., Robert, K. H. and Sundqvist, K-G. (1993). Chemotaxis and haptotaxis of human malignant mesothelioma cells, Effects of fibronectin, laminin, type IV collagen, and an autocrine motility factor-like substance. Cancer Res, 53, $4376-4382$

Koshiba, T., Hosotani, R., Wada, M., Miyamoto, Y., Fujimoto, K., Lee, J.-U., Doi, R., Arii, S. and Inamura, M. (1998). Involvement of matrix metalloproteinases-2 activity in invasion and metastasis of pancreatic carcinoma. Cancer, 82, 642-650.

Lacovara, J., Cramer, E. B. and Quigley, J. P. (1984). Fibronectin enhancement of directed migration of B16 melanoma cells. Cancer Res., 44, 1657-1663.

Lawrence, J. A. and Steeg, P. S. (1996). Mechanisms of tumour invasion and metastasis. World J. Urol, 14, 124-130.

Liotta, L. A., Rao, C. N. and Barsky, S. H. (1983). Tumour invasion and the extracellular matrix. Lab. Invest, 49, 636-649.

McCarthy, J. B. and Furcht, L. T. (1984). Laminin and fibronectin promote the directed migration of B 16 melanoma cells in vitro. J. Cell Biol, 98, 1474-1480.

Marusic, M., Bajzer, Z., Freyer, J. P. and Vuk-Pavlovic, S. (1994). Analysis of growth of multicellular tumour spheroids by mathematical models. Cell Prolif, 27, 73-94.

Matrisian, L. M. (1992). The matrix-degrading metalloproteinases. Bioessays, 14, 455-463.

Mignatti, P. and Rifkin, D. B. (1993). Biology and biochemistry of proteinases in tumor invasion. Physiol. Rev, 73, 161-195.

Mitchell, A. R. and Griffiths, D. F. (1980). The finite difference method in partial differential equations, Wiley, Chichester.

Orme, M. E. and Chaplain, M. A. J. (1996). A mathematical model of vascular tumour growth and invasion. Mathl. Comp. Modelling, 23, 43-60.

Othmer, H. and Stevens, A. (1997). Aggregation, blowup and collapse, The ABCs of taxis and reinforced random walks. SIAM J. Appl. Math, 57, 1044-1081.

Parson, S. L., Watson, S. A., Brown, P. D., Collins, H. M. and Steele, R. J. C. (1997). Matrix metalloptoteinases. Brit. J. Surg, 84, $160-166$.

Perumpanani, A. J., Sherratt, J. A., Norbury, J. and Byrne, H. M. (1996). Biological inferences from a mathematical model of malignant invasion. Invasion and Metastases, 16, 209-221.

Qi, A., Zheng, X., Du, C. and An, B. (1993). A Cellular Automaton Model of Cancerous Growth. J. theor. Biol, 161, $1-12$. 
Quigley, J. P., Lacovara, J. and Cramer, E. B. (1983). The directed migration of B-16 melanoma-cells in response to a haptotactic chemotactic gradient of fibronectin. J. Cell Biol, 97, A 450-451.

Retsky, M. W., Swartzendruber, D. E., Wardwell, R. H. and Bame, P. D. (1990). Is gompertzian or exponential kinetics a valid description of individual human cancer growth? Medical Hypotheses, 33, 95-106.

Sehgal, G., Hua, J., Bernhard, E. J., Sehgal, I., Thompson, T. C. and Muschel, R. J. (1998). Requirement for matrix metalloproteinases- 9 (gelatinase B) expression in metastasis by murine prostate carcinoma. Am. J. Pathol, 152, 591-596.

Sherratt, J. A. and Nowak, M. A. (1992). Oncogenes, antioncogenes and the immune response to cancer, a mathematical model. Proc. R. Soc. Lond. B, 248, 261-271.

Smolle, J. and Stettner, H. (1993). Computer simulation of tumour cell invasion by a stochastic growth model. $J$, theor. Biol, 160, $63-72$.

Smolle, J. and Grimstad, I. A. (1992). Tumor-cell motility and invasion within tumours determined by applying computer simulation to histologic patterns. Int. J. Cancer, 50, 331-335.

Smolle, J., Soyer, H. P., Smolle-Juettner, F. M., Stettner, H. and Kerl, H. (1990). Computer simulation of tumour cell motility and proliferation. Path. Res. Pract, 186, 467-472.

Stetler-Stevenson, W. G., Krutzach, H. L. and Liotta, L. A. (1989). Tissue inhibitor of metalloproteinases (TIMP-2). J. Biol. Chem, 264, 17372-17378.
Stetler-Stevenson, W. G., Aznavoorian, S. and Liotta, L. A. (1993). Tumor cell interactions with the extracellular matrix during invasion and metastasis. Ann. Rev. Cell Biol, 9, $541-573$.

Stetler-Stevenson, W, G., Hewitt, R. and Corcoran, M. (1996). Matrix metallo-proteinases and tumour invasion, from correlation to causality to the clinic. Cancer Biol, 7, 147-154.

Terranova, V. P., Difforio, R., Lyall, R. M., Hic, S., Friesel, R. and Maciag. T. (1985). Human endothelial cells are chemotactic to endothelial cell growth factor and heparin. J. Cell Biol, 101, $2330-2334$.

Thorgeirsson, U. P., Lindsay, C. K., Cottam, D. W. and Daniel, Gomez, E. (1994). Tumor invasion, proteolysis, and angiogenesis. J. Neuro-Oncology, 18, 89-103.

Tracqui, P. (1995). From passive diffusion to active cellular migration in mathematical models of tumour invasion. Acta Biotheor, 43, $443-464$.

Wheldon, T. E. (1986). Mathematical models in experimental and clinical oncology. In: Mathematical Methods in Medicine, 1-32, D. Ingram and R.F. Bloch Eds, London, J. Wiley and Sons.

Yu, A. E., Murphy, A. N. and Stetler-Stevenson, W. G. (1998). Gelatinase A. In Matrix Metalloproteinases (W.C. Parks, R.P. Meecham eds.), pp. 85-113. Academic Press, San Diego.

Zeng, Z. S. and Guillem, J. G. (1998). Unique activation of matrix metalloproteinase-9 within human liver metastasis from colorectal cancer. Brit. J. Cancer, 78, 349-353. 


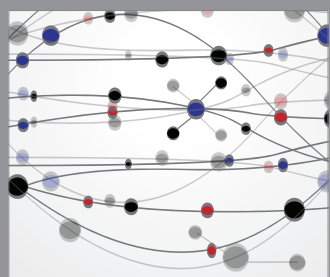

The Scientific World Journal
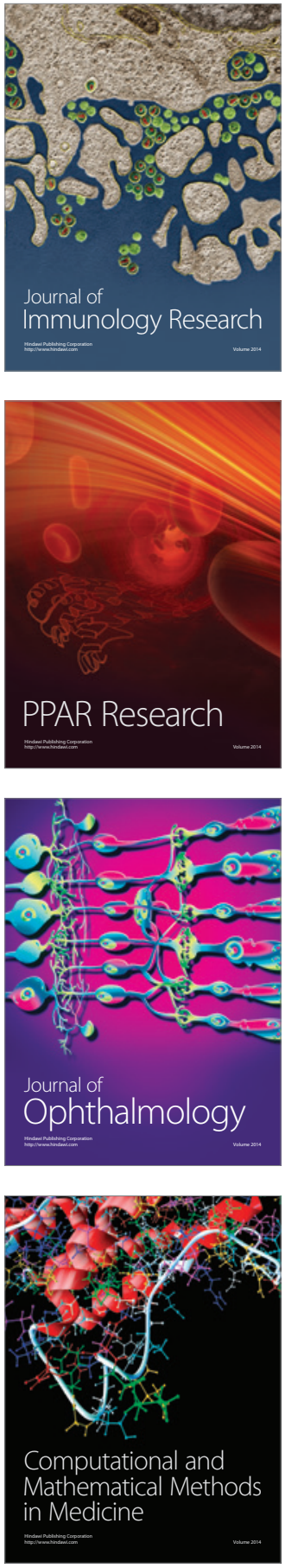

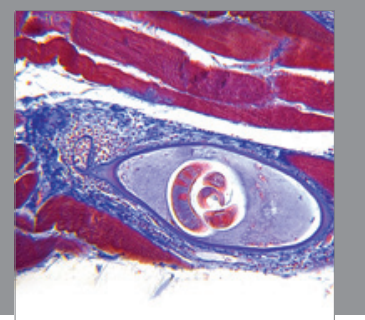

Gastroenterology

Research and Practice
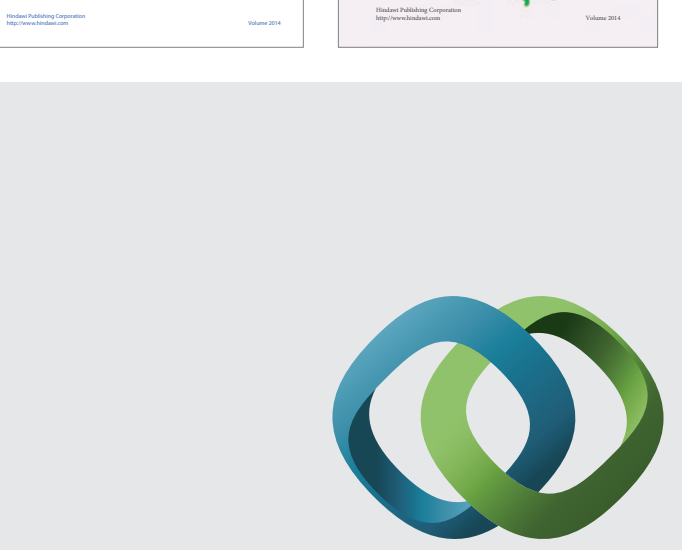

\section{Hindawi}

Submit your manuscripts at

http://www.hindawi.com
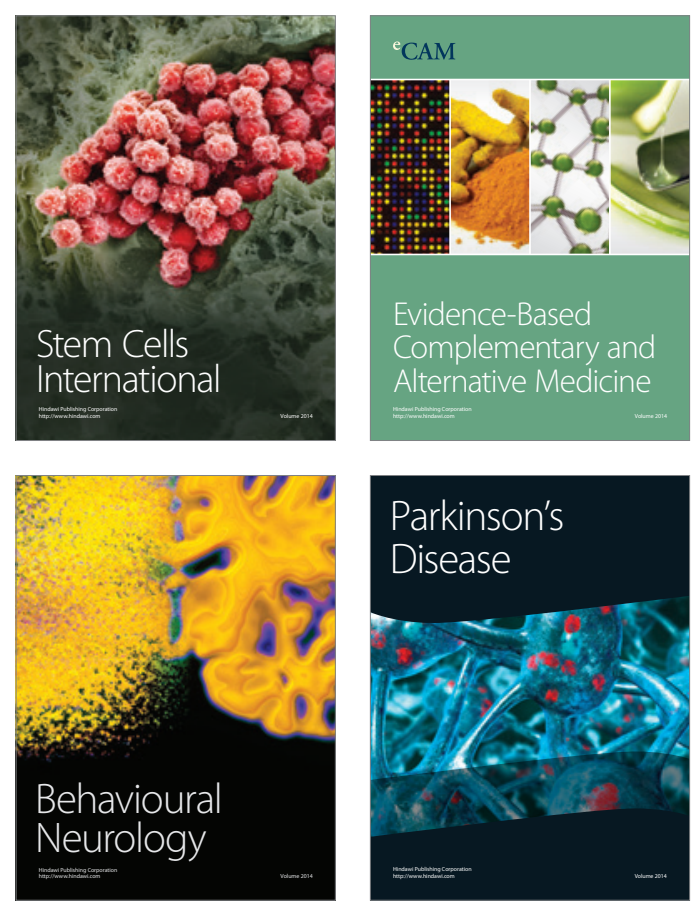

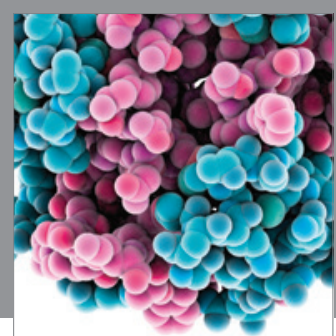

Journal of
Diabetes Research

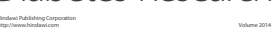

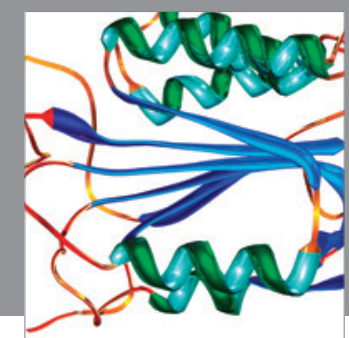

Disease Markers
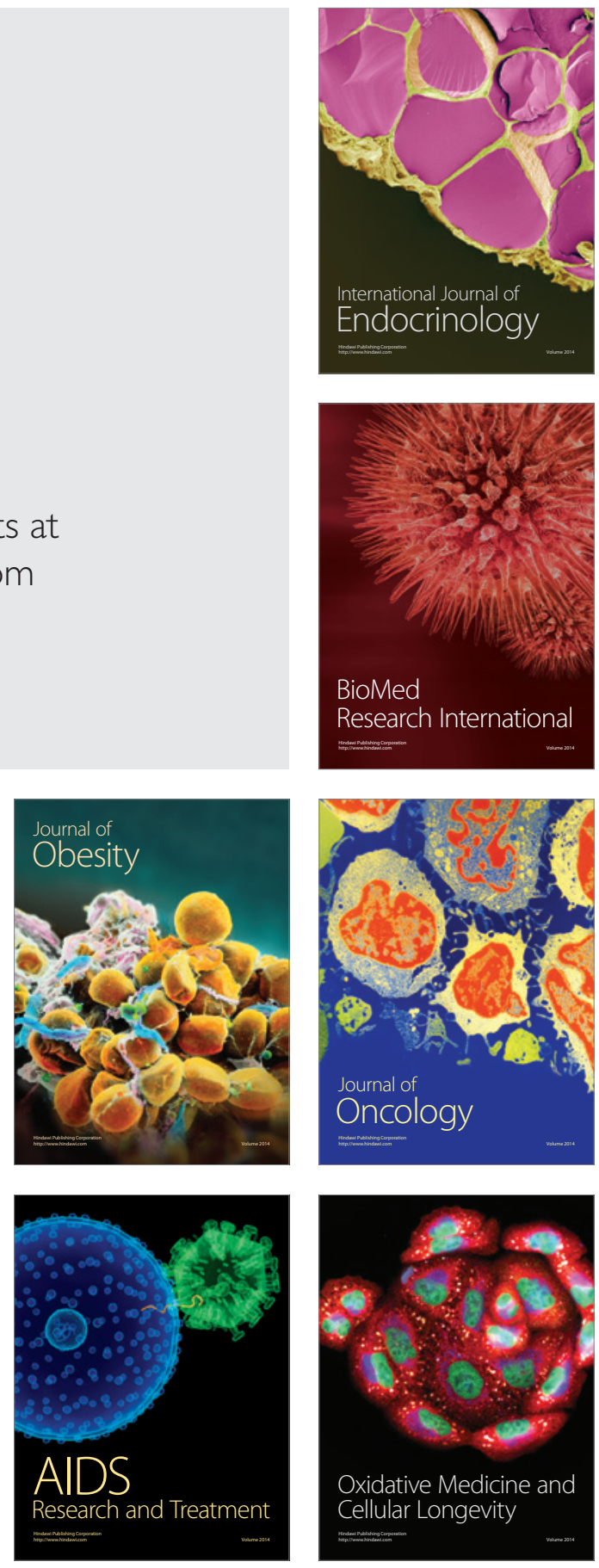